\title{
Beam and spin dynamics in the fast ramping storage ring ELSA Concepts and measures to increase beam energy, current and polarization
}

\author{
Wolfgang Hillert ${ }^{1, \star}$, Andreas Balling ${ }^{2}$, Oliver Boldt ${ }^{2}$, Andreas Dieckmann ${ }^{1}$, Maren Eberhardt ${ }^{2}$, Frank Frommberger ${ }^{1}$, \\ Dominik Heiliger ${ }^{3}$, Nikolas Heurich ${ }^{1}$, Rebecca Koop ${ }^{1}$, Fabian Klarner ${ }^{2}$, Oliver Preisner ${ }^{3}$, Dennis Proft ${ }^{1}$, Thorsten \\ Pusch $^{4}$, André Roth ${ }^{2}$, Dennis Sauerland ${ }^{1}$, Manuel Schedler ${ }^{5}$, Jan Felix Schmidt ${ }^{1}$, Michael Switka ${ }^{1}$, Jens-Peter \\ Thiry $^{1}$, Jürgen Wittschen ${ }^{2}$, and Sven Zander ${ }^{6}$ \\ ${ }^{1}$ University of Bonn, Physics Institute, ELSA, Nussallee 12, D-53115 Bonn, Germany \\ ${ }^{2}$ Varian Medical Systems Particle Therapy GmbH, Mottmannstr. 2, D-53842 Troisdorf, Germany \\ ${ }^{3}$ TÜV Rheinland Industrie Service GmbH, Am Grauen Stein, D-51105 Köln, Germany \\ ${ }^{4}$ Fraunhofer-Institut für Naturwissenschaftlich-Technische Trendanalysen, Appelsgarten 2, D-53879 Euskirchen, Germany \\ ${ }^{5}$ Karlsruhe Institute of Technology, LAS, Kaiserstraße 12, D-76131 Karlsruhe, Germany \\ ${ }^{6}$ TÜV Nord EnSys GmbH \& Co. KG, Große Bahnstraße 1, D-22525 Hamburg, Germany
}

\begin{abstract}
The electron accelerator facility ELSA has been operated for almost 30 years serving nuclear physics experiments investigating the sub-nuclear structure of matter. Within the 12 years funding period of the collaborative research center SFB/TR 16 , linearly and circularly polarized photon beams with energies up to more than $3 \mathrm{GeV}$ were successfully delivered to photoproduction experiments. In order to fulfill the increasing demands on beam polarization and intensity, a comprehensive research and upgrade program has been carried out. Beam and spin dynamics have been studied theoretically and experimentally, and sophisticated new devices have been developed and installed. The improvements led to a significant increase of the available beam polarization and intensity. A further increase of beam energy seems feasible with the implementation of superconducting cavities.
\end{abstract}

\section{Introduction}

Accelerator research, development and operation has been performed at the University of Bonn for more than 60 years. In the beginning a small $500 \mathrm{MeV}$ strong focussing synchrotron provided the first electron beam in 1958. A larger fast cycling $2.5 \mathrm{GeV}$ alternating gradient synchrotron was constructed and is in operation since 1967. It was extended in 1987 by the electron stretcher and $3.5 \mathrm{GeV}$ storage ring ELSA, leading to increased energy and improved beam characteristics. The complete accelerator facility (see Fig. 1) is based on the storage ring and an injector chain consisting of two linear accelerators and the fast cycling booster synchrotron [1].

Within the collaborative research center SFB/TR16, which has been established in 2004, ELSA is serving two photoproduction experiments (CBELSA/TAPS [2] and BGO-OD [3]) with a quasi-continuous electron beam. The external electron beam is generated via slow resonance extraction from the storage ring over a periodically recurring time frame of a few seconds and converted to a photon beam via the process of bremsstrahlung. The energy of the photons is determined by dedicated tagger spectrometers that measure the energy of those electrons which radiated a photon $[2,4]$.

\footnotetext{
^e-mail: hillert@physik.uni-bonn.de
}

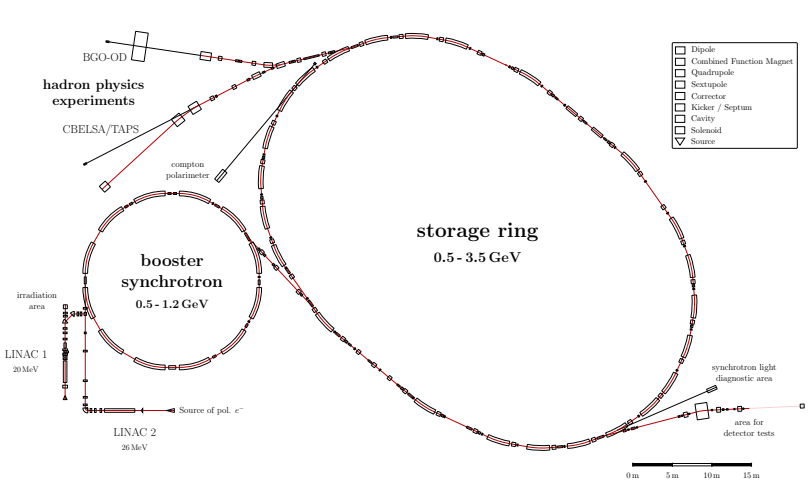

Figure 1. The accelerator facility ELSA at Bonn University.

The experimental program pursued in the SFB/TR 16 is based on the availability of linearly and circularly polarized photons. Whereas linearly polarized photons are produced by coherent bremsstrahlung off a diamond crystal [5], circularly polarized photons are generated by polarization transfer from longitudinally polarized electrons in the bremsstrahlung process.

Therefore both experimental setups demand a stable and reliable delivery of an electron beam with sufficient intensity, polarization and energy. Over the past 12 years, intense accelerator research and development has been car- 
ried out to increase the degree of beam polarization and intensity as well as to enhance the stability of the external electron beam. In addition, several aspects of a maximum beam energy increase to $5 \mathrm{GeV}$ have been investigated. In this paper we report on the related upgrade program of the ELSA accelerator and present the results obtained.

\section{Polarized electrons}

Polarized electrons are generated in a dedicated source [6] which has been developed and set up primarily for the measurement of the Gerasimov-Drell-Hearn sum rule [79]. In order to enhance the uptime of the source, the existing load-lock has been extended, implementing a newly developed photocathode surface cleaning procedure using atomic hydrogen [10-13]. By optimizing the optical settings of the transfer line to the linear accelerator, the available beam current has been increased significantly $[11,14]$.

Beam depolarization due to crossing of depolarizing resonances on the fast energy ramp in the storage ring has been successfully suppressed by different measures [15]. A newly developed orbit correction system has been implemented, allowing for a fast setting of harmonic corrections [16-20]. In combination with a novel harmonic spin-orbit correction scheme, integer depolarizing resonances have been effectively compensated [21, 22]. Intrinsic depolarizing resonances are routinely suppressed by increasing the crossing speed utilizing pulsed tune jump quadrupole magnets [23].

Depolarizing effects have been investigated theoretically using numerical simulation techniques [24-27]. A dedicated simulation tool polematrix has been developed which allows detailed studies of beam depolarization when crossing depolarizing resonances [27].

The measurement of the polarization degree (polarimetry) is typically performed using a Møller polarimeter in the external beam line to the CBELSA/TAPS experiment. In order to directly measure the polarization of the stored electron beam, a Compton polarimeter has been set up at the storage ring [28-30]. First measurements show the expected build-up of polarization caused by the Sokolov-Ternov mechanism.

\subsection{Source of polarized electrons}

Polarized electrons are produced by irradiating a strainedlayer superlattice photocathode with circularly polarized laser light from a flashlamp pumped Ti:sapphire laser. In order to enable the electrons to escape into vacuum, the photocathode has to be activated first by cleaning the surface from remaining contaminations and thereafter depositing a monomolecular layer of cesium and oxygen. Due to the adsorption of contaminating molecules the initial quantum efficiency $Q E_{0}$ after an activation procedure decreases with time $t$ according to

$$
Q E(t)=Q E_{0} \cdot \exp \left(-\frac{t}{\tau}\right)
$$

The time constant $\tau$ is called the lifetime of the photocathode and is strongly dependent on the quality of the vacuum. It is therefore indispensable to achieve a total pressure of $10^{-11}$ mbar and partial pressures of the most contaminating gases like water vapor, oxygen and carbon dioxide in the range of $10^{-13}$ mbar in the operating chamber of the source. This extreme ultrahigh vacuum (XHV) has to be preserved by an effective isolation from the moderate vacuum of the subsequent linear accelerator. Beam loss in the transfer line will cause additional gas desorption and has to be prevented by careful beam steering and focussing, based on numerical simulations of the beam dynamics. Insertion of new photocathodes as well as heat cleaning and activation of used photocathodes require additional vacuum chambers and a transfer to the operating chamber without breaking the XHV conditions. Such a load-lock system has been installed at the operating chamber becoming an integral part of the source.

\subsubsection{Beam transport}

For the first years of operation, the beam current in standard operation was set to $100 \mathrm{~mA}$. After changing from a Be-InGaAs/Be-AlGaAs to a GaAs/GaAsP photocathode and increasing the usable cathode diameter from $8 \mathrm{~mm}$ to $8.9 \mathrm{~mm}$, a considerably higher emission current of up to $200 \mathrm{~mA}$ has been produced reliably but not safely transported to the linac. To benefit from this increased intensity, numerical simulations of the beam transport have been carried out to derive optimal settings of the focussing elements of the transfer line for minimizing beam loss. The experimental optimization has been performed by additionally detecting the transverse beam intensity profiles using dedicated wire scanners located at different positions in the transfer line.

\section{Transfer line}

The transfer line connects the operation chamber with the linear accelerator (see Fig. 2).

Along the entire transfer line, 41 magnets are installed. There are two symmetry planes: one at the second wire scanner and one at the Mott polarimeter. The most important elements of the folded beam line are an electrostatic deflector and two $\alpha$-magnets for deflecting as well as solenoids and quadrupoles for the focussing of the beam.

To ensure a conservation of polarization in the following circular accelerators, the longitudinal spin is rotated into the transverse plane with respect to the momentum by the electrostatic deflector. To avoid a further influence on the spin orientation, mainly double solenoids are used to focus the beam behind the deflector. The polarization is measured using a Mott polarimeter.

\section{Beam dynamics and simulation}

The transversal beam dynamics of a low-energy electron beam with a homogeneous, elliptical charge distribution ${ }^{1}$

\footnotetext{
${ }^{1}$ The so called Kapchinsky-Vladimirsky distribution is assumed, whose projection is a sharp-edged, homogeneous and elliptical charge distribution [31].
} 


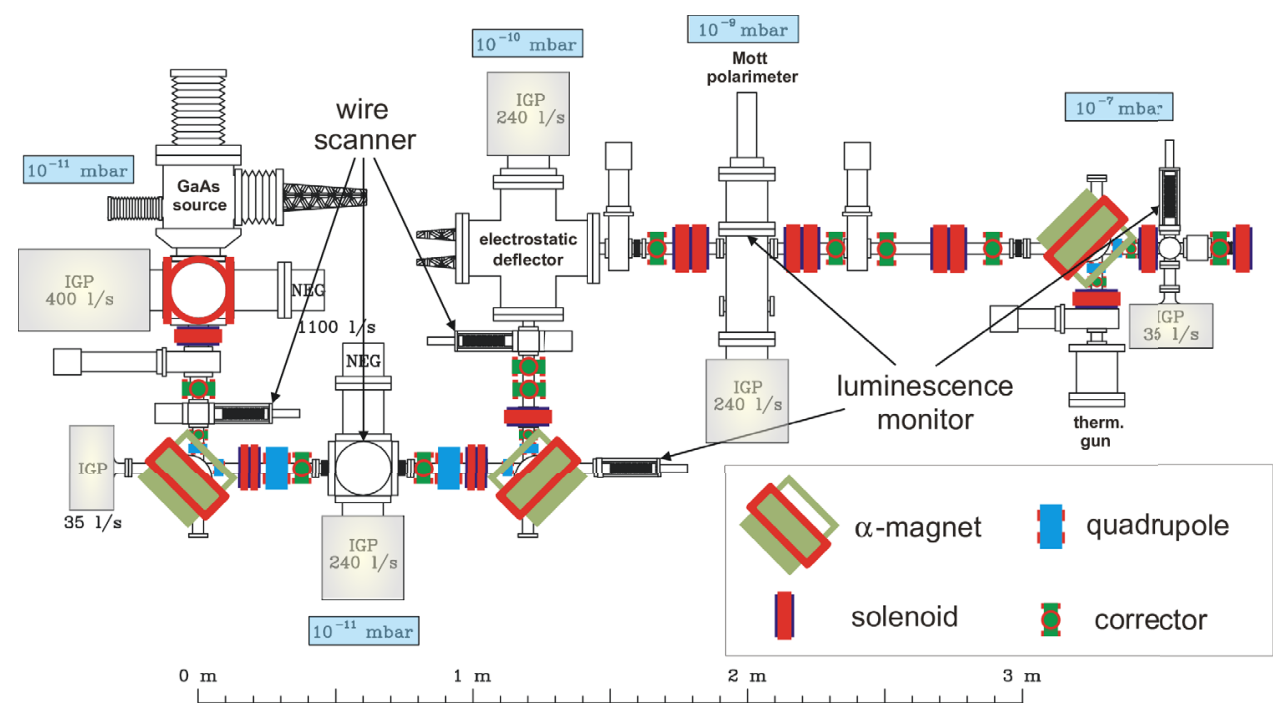

Figure 2. The transfer line between the operating chamber and the linear accelerator [10].

in presence of external electromagnetic fields assuming a "laminar" flow are described by the so called paraxial differential equations [31]:

$$
\begin{aligned}
& \frac{\mathrm{d}^{2} x}{\mathrm{~d} s^{2}}+\left[k_{x}(s)+S(s)+T(s)\right] \cdot x-\frac{\varepsilon^{2}}{x^{3}}-\frac{2 K}{x+z}=0 \\
& \frac{\mathrm{d}^{2} z}{\mathrm{~d} s^{2}}+\left[k_{z}(s)+S(s)+T(s)\right] \cdot z-\frac{\varepsilon^{2}}{z^{3}}-\frac{2 K}{x+z}=0 .
\end{aligned}
$$

The increase of the beam envelope due to the emittance $\varepsilon$ and the space charge are expressed in the third and fourth term. $K$ is called the generalized perveance and depends on the beam current and energy. In the first three terms the restoring forces of quadrupoles $\left(k_{x, z}(s)\right)$, solenoids $(S(s))$ and the electrostatic deflector $(T(s))$ are included.

Due to the low beam energy and the high intensity, the beam transport is strongly space charge dominated. Hence, all focal lengths of the magnets have to be adjusted according to the actual beam current. To examine the feasibility of a beam transport of $200 \mathrm{~mA}$ using the existing magnets in the transfer line, the focussing strengths of the magnets were optimized by iteratively feeding adjusted parameter sets into an in-house developed software package used for the numerical integration of the differential equations. The optimization criteria were a beam focus in the symmetry planes and a minimal beam envelope along the whole transfer line in order to approach a lossless beam transport to the linear accelerator and not to degrade the vacuum.

The final results of the simulations for currents of $100 \mathrm{~mA}$ and $200 \mathrm{~mA}$ are shown in Fig. 3. The solid lines indicate the beam envelope for the optimal settings of the focussing strengths of the magnets. The initial point of the simulation is the first beam waist close to the anode, whose position varies for different currents and diameters of the emitting surface and is caused by the focussing geometry of the electrodes. The origin of the coordinate system was set to the position of this beam waist for $100 \mathrm{~mA}$. The initial parameters, such as the beam envelope at the waist

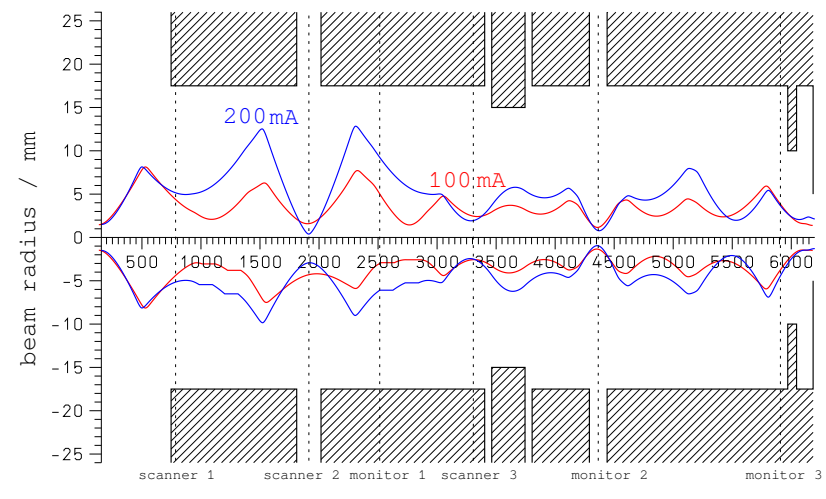

Figure 3. Final results of the numerical simulations for a beam current of $100 \mathrm{~mA}$ (diameter of the photocathode $\varnothing_{\mathrm{pc}}=8.0 \mathrm{~mm}$ ) and $200 \mathrm{~mA}\left(\varnothing_{\mathrm{pc}}=8.9 \mathrm{~mm}\right)$. The optimization criterion of a minimum beam envelope at the symmetry points is fulfilled for both currents [10].

and the position of the waist as well as the emittance, were numerically simulated for each emission current using the software EGUN[32].

Even in case of a current of $200 \mathrm{~mA}$ the radius is always smaller than one half of the aperture except near the $\alpha$-magnets, indicating that a quasi lossless transport appears to be feasible with $200 \mathrm{~mA}$.

\section{Achieved performance}

To experimentally optimize the optics of the transfer line, measurements of the beam profiles were performed under variation of the focussing strength of the magnets based on the simulation results for a current of $200 \mathrm{~mA}$. The adjustments for a current of $100 \mathrm{~mA}$ were used as initial values.

The two beam profiles shown in Fig. 4 were recorded with the first wire scanner in the transfer line at a beam current of $200 \mathrm{~mA}$. The measurements are in reasonable 


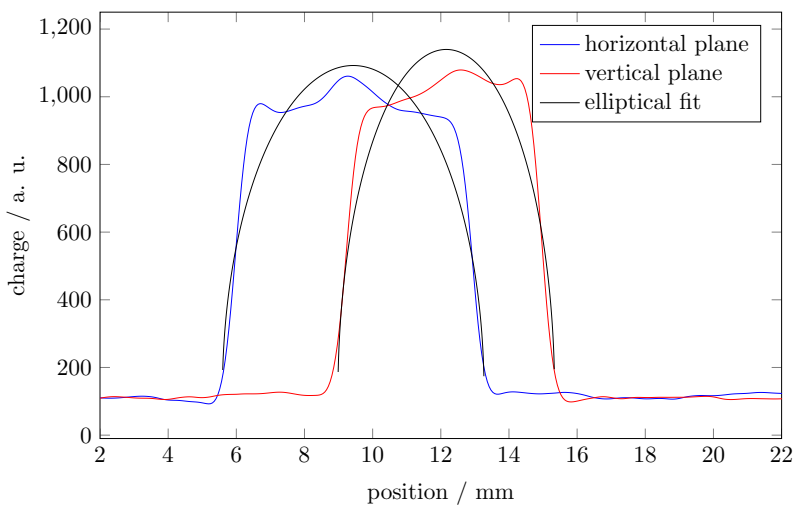

Figure 4. Measurement of the beam profile with the first wire scanner and a beam current of $200 \mathrm{~mA}$ [33].

agreement with a fitted elliptical profile expected for a homogeneous cylinder symmetric charge distribution. So the assumptions made for the paraxial differential equations seem justified. In order to ensure an elliptical charge distribution also at the end of the transfer line, beam profile measurements were performed with the third wire scanner. Figure 5 shows this measurement at a beam current of $200 \mathrm{~mA}$ proving the expected beam profile. The slight charge redistribution visible in Fig. 4 and 5 can be explained by an inhomogeneous fringe field of a permanent magnet belonging to an ion getter pump.

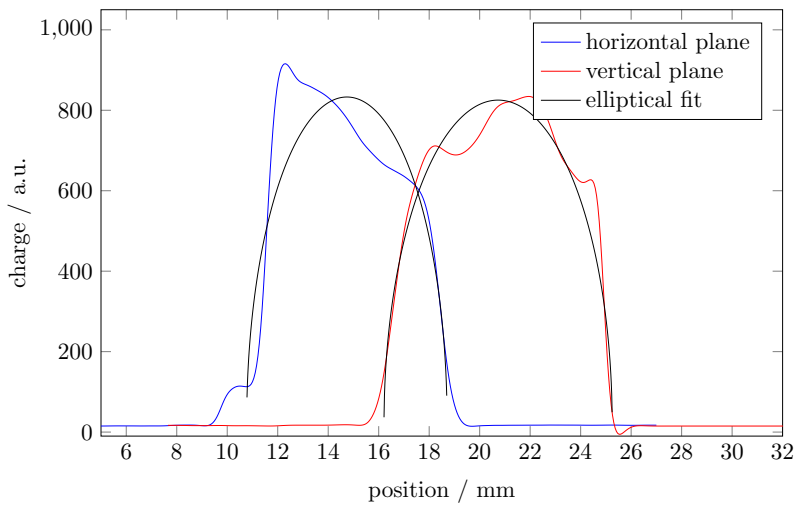

Figure 5. Measurement of the beam profile with the third wire scanner and a beam current of $200 \mathrm{~mA} \mathrm{[33].}$

The beam profile measurements verify the assumption of a nearly cylinder symmetric, homogeneous and sharpedged beam profile over the whole transfer line. Finally, an optimized optics setting of the transfer line with an overall beam loss of less than $1 \%$ for beam currents of almost $200 \mathrm{~mA}$ has been determined experimentally.

\subsubsection{New load-lock system}

\section{Overview}

The new load-lock system consists of three XHV chambers (see Fig. 6). The loading chamber allows the insertion

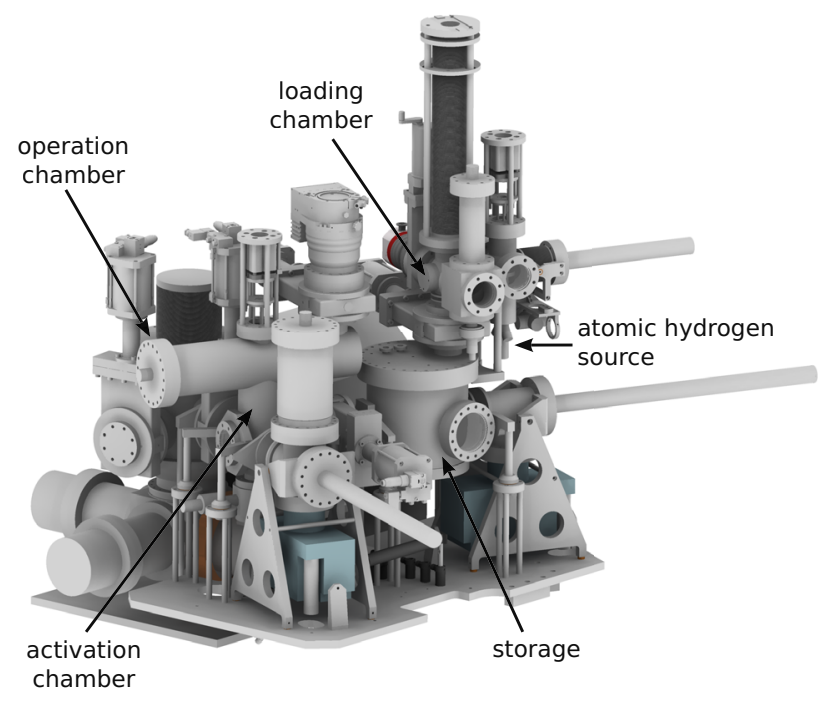

Figure 6. Overview of the new load-lock system with its three XHV chambers [13].

of a new photocathode and cleaning it with atomic hydrogen for a better removal of surface oxidations. The photocathode can be transported to the storage chamber via an elevator, where several photocathodes can be stored under extreme high vacuum conditions. In the activation chamber the photocathode is prepared for operation by evaporating cesium and oxygen on its surface. All chambers were baked out for two weeks to reduce the desorption of molecules from the walls and achieve the XHV.

\section{Storage chamber}

In the storage chamber XHV conditions (total pressure $5 \times 10^{-12} \mathrm{mbar}$ ) are achieved by four NEG modules $(330 \mathrm{~L} / \mathrm{s}$ each) and an ion getter pump $(75 \mathrm{~L} / \mathrm{s})$. Photocathodes can be stored inside the chamber on a rotary plate (see Fig. 7), which is adjustable in height by a bellow. The force transmission between the vacuum and non-vacuum side is carried without contact by magnets.

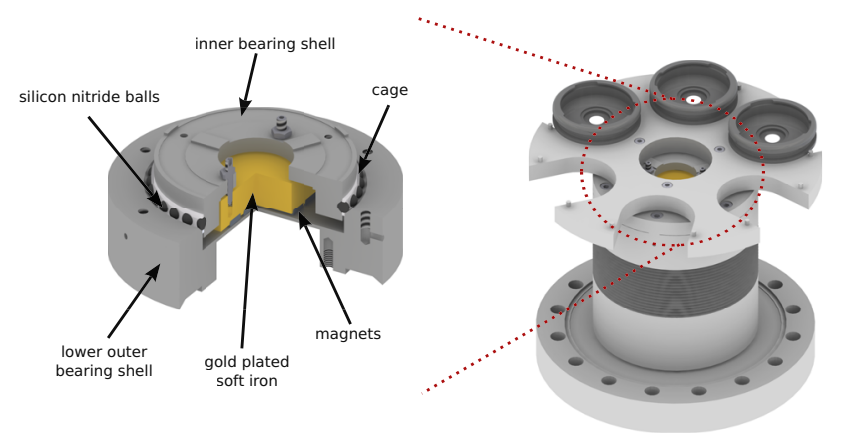

Figure 7. The rotary plate in the storage chamber and a cut through the ball bearing [33].

After one year of pumping resulting in a high degree of surface purification and possible cold shut of the materials, the ball bearing of the rotary plate moved tightly. As a precaution the rotary plate was clamped in a fixed 


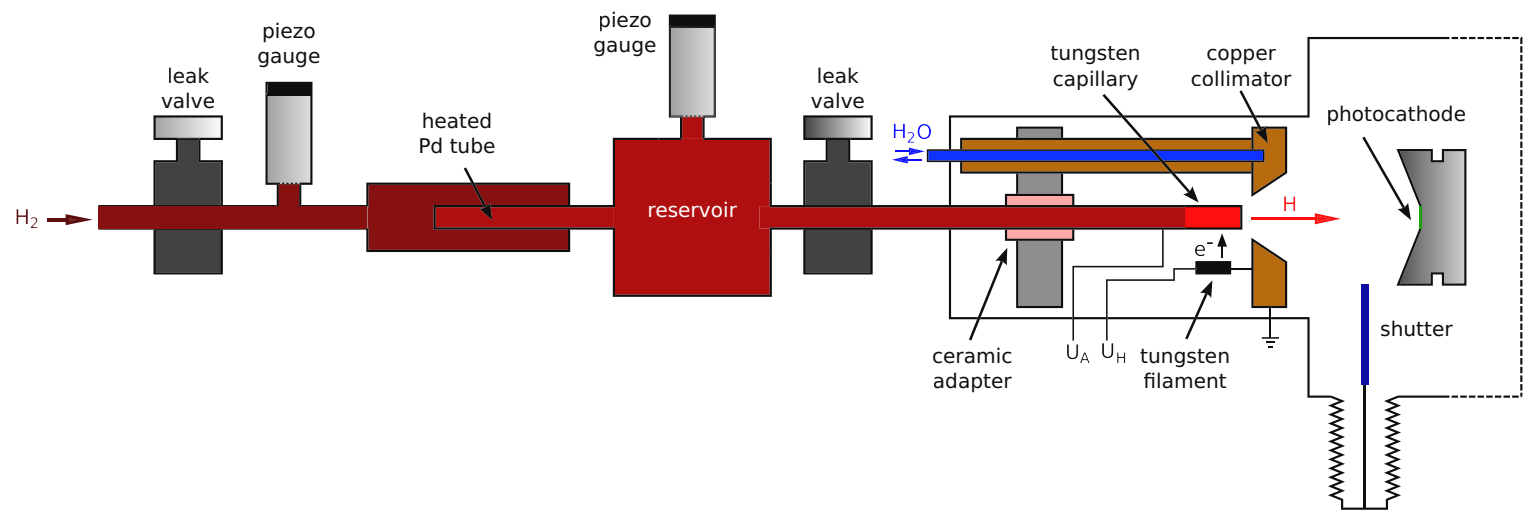

Figure 8. Inlet of hydrogen into the loading chamber for H-cleaning [13].

position to ensure that at least two storage areas are accessible. Further investigations have to be performed in order to understand the exact mechanism and to guarantee a long time low friction movement of the rotary plate. Nevertheless up to three photocathodes can be stored and used for operation within the new load-lock system even if the rotary plate is fixed.

\section{Activation chamber}

In this chamber a photocathode can be heat cleaned and activated with cesium and oxygen. An ion getter pump (75 L/s), two NEG-pumps $(2000 \mathrm{~L} / \mathrm{s}$ each) and a turbo molecular pump $(300 \mathrm{~L} / \mathrm{s})$ provide an ultra high vacuum during these processes. By alternately heating a silver tube $^{2}$ and cesium dispensers, a sub-mono molecular layer of CsO-dipoles is evaporated on the surface of the photocathode. The quantum efficiency and the emitted current during the activation process is measured by irradiating the photocathode with laser light from a helium-neon laser and applying an accelerating voltage between the photocathode and the ground potential. A countervoltage within the distance of acceleration prevents a backstreaming of ions evaporated from the cesium dispensers.

For heat cleaning two cylindrical radiation shields with a heated filament encase the photocathode and heat it by thermal radiation up to $550^{\circ} \mathrm{C}$. Most of the oxidations desorb from the photocathode's surface at this temperature, but especially strongly ligated molecules like galliumoxides do not leave the photocathode's surface below its damage threshold of about $600{ }^{\circ} \mathrm{C}$. Thus, the quantum efficiency does not reach its initial value after the cleaning and decreases over several activations until the photocathode can no longer be used for operation, because not all impurities are removed. Additionally, heat cleaning produces a rough surface, which reduces the optimal orientation of the CsO-dipoles, the quantum efficiency as well as the polarization degree $[34,35]$. Due to these disadvantages a source for atomic hydrogen was installed inside the loading chamber.

\footnotetext{
${ }^{2}$ Heated silver is permeable for oxygen only.
}

\section{Loading chamber}

The loading chamber fulfills two main functions: it allows the insertion of new photocathodes and the cleaning of the photocathode's surface by thermal heating with additional supply of atomic hydrogen.

Atomic hydrogen converts strongly ligated molecules (like $\mathrm{Ga}_{2} \mathrm{O}_{3}, \mathrm{Ga}_{2} \mathrm{O}$ and $\mathrm{As}_{2} \mathrm{O}_{x}$ ) on the photocathode's surface, that are resistant to heat cleaning, into volatile ones $[34,36,37]$ :

$$
\begin{aligned}
\mathrm{As}_{2} \mathrm{O}_{x}+2 x \mathrm{H} & \rightarrow \mathrm{As}_{2}+x \mathrm{H}_{2} \mathrm{O} \uparrow \\
\mathrm{Ga}_{2} \mathrm{O}+2 \mathrm{H} & \rightarrow 2 \mathrm{Ga}+\mathrm{H}_{2} \mathrm{O} \uparrow \\
\mathrm{Ga}_{2} \mathrm{O}_{3}+4 \mathrm{H} & \rightarrow \mathrm{Ga}_{2} \mathrm{O}+2 \mathrm{H}_{2} \mathrm{O} \uparrow .
\end{aligned}
$$

The arrows in the equations above indicate the oxidations (ligated as $\mathrm{H}_{2} \mathrm{O}$ ), which are removed by additionally heating the photocathode to around $550{ }^{\circ} \mathrm{C}$. The gallium and arsenic reaction products remain on the surface. The atomic hydrogen beam does not contain any hydrogen ions, because of thermal cracking. This results in an atomically clean and flat surface with only a few defects near to the surface $[35,38]$.

\section{Hydrogen filtering, inlet and cracking}

A maximum degree of purity of hydrogen is desired for the cleaning procedure. Thus, besides using highly purified molecular hydrogen $(99.9999 \%)$ from a gas bottle, an additional filter is utilized. It consists of a palladium tube $(5 \mathrm{~cm}$ in length, inner and outer diameter of $5 \mathrm{~mm}$ and $6 \mathrm{~mm}$ ). Heated palladium above temperatures of $200^{\circ} \mathrm{C}$ is permeable for hydrogen. The tube is heated using a wire with high oxidation resistance, the temperature is measured with a thermocouple. Both sides of the tube can be evacuated before and after hydrogen cleaning, in order to prevent the formation of a hydride phase while cooling the tube below $150^{\circ} \mathrm{C}$ in presence of hydrogen [39]. The inner side of the tube is clean and evacuated anyway, because it was baked out together with the loading chamber.

The filtered hydrogen accumulates on the baked-out vacuum side of the tube in a reservoir, where the pressure is measured with a piezo gauge. Afterwards the hydrogen is piped through a capillary, which is heated by electron 
bombardment up to $1900{ }^{\circ} \mathrm{C}$ [40] controlling the flow rate with a calibrated leak valve. In this capillary the thermal energy cracks the molecular hydrogen into atomic hydrogen with a high dissociation rate (see Fig. 8). The photocathode is heated to $550{ }^{\circ} \mathrm{C}$ while atomic hydrogen cleaning is applied to assure a rapid evaporation of the volatile reaction products, mainly $\mathrm{H}_{2} \mathrm{O}$. A turbo molecular pump and a NEG-pump keep the pressure during the hydrogen inlet below $3 \times 10^{-6}$ mbar.

Several measurements with different photocathodes demonstrated the success of this cleaning procedure (see [33]).

\subsection{Acceleration of polarized electrons}

Polarized electrons are accumulated in the ELSA storage ring via the $1.2 \mathrm{GeV}$ booster synchrotron, then accelerated utilizing a fast energy ramp with up to $6 \mathrm{GeV} / \mathrm{s}$ and delivered to the experiments via slow extraction. This concept requires to preserve the polarization during the whole acceleration process.

During acceleration, the crossing of several depolarizing resonances may cause severe beam depolarization. Even in case of fast ramping speeds of up to $6 \mathrm{GeV} / \mathrm{s}$, first order effects like integer and intrinsic resonances have to be compensated by dedicated measures [24]. Depolarizing resonances occur whenever the electron spin precession around the vertical fields of the bending magnets is in phase with any component of the accelerator's horizontal magnetic field distribution. According to the ThomasBMT equation [41], the spins precess $\gamma a$ times per turn in a flat circular electron accelerator (neglecting longitudinal magnetic fields), where $a=\left(g_{s}-2\right) / 2$ is the gyromagnetic anomaly. $\gamma a$ is also called the spin tune and increases linearly with beam energy due to the Lorentz factor $\gamma$. Several depolarizing resonances are crossed during the acceleration in the ELSA storage ring. Their correction is the major challenge to reach high degrees of polarization. Without any compensation all polarization might be lost at $2.35 \mathrm{GeV}$. Disabling all dedicated countermeasures merely up to $30 \%$ can be reached.

There are two main types of depolarizing resonances. Integer spin tune resonances occur about every $440 \mathrm{MeV}$, when the spin tune $\gamma a$ becomes an integer number. Accordingly, these resonances are driven by those horizontal magnetic fields that act on the beam in the same way each turn. In other words, the resonance $\gamma a=n$ is driven by the field component changing with the $n$th revolution harmonic around the ring. These are e.g. magnet misalignments as well as quadrupole fields caused by a vertical closed orbit displacement. Thus, the first measure against integer resonances is a preferably good vertical closed orbit correction during the fast energy ramp (see Section 2.2.1). Additionally, the remaining depolarizing field component has to be compensated.

In proton accelerators, usually integer resonances are deliberately excited to induce a full spin flip during resonance crossing and preserve the absolute value of polarization. This is not possible for electron beams, because of

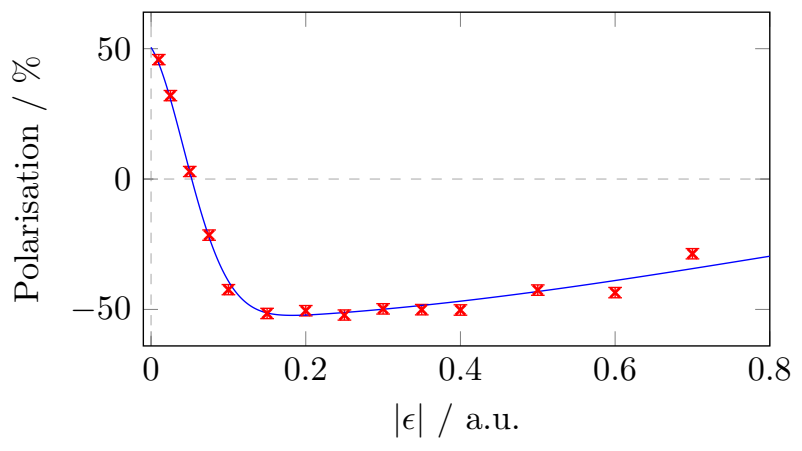

Figure 9. Measurement of the Froissart-Stora curve for the integer resonance $\gamma a=5$ in the ELSA storage ring.

synchrotron radiation. The radiative energy loss requires permanent accelerating rf fields leading to higher synchrotron frequencies compared to proton beams. Therefore, the synchrotron sidebands of depolarizing resonances have a larger distance from the central resonance and are crossed separately during the energy ramp. This results in additional depolarization and a complete spin flip is not possible according to the Froissart-Stora formula [42]. The measurement shown in Fig. 9 illustrates this effect. Here, the polarization after crossing the integer resonance $\gamma a=5$ in the ELSA storage ring is shown depending on the resonance strength $\epsilon$. It was measured with the Møller polarimeter at the CBELSA/TAPS experiment. The resonance strength was varied using the vertical orbit correction magnets. Because the absolute value of polarization decreases for high resonance strengths, integer resonances are compensated at ELSA by minimizing the resonance strength with a properly adjusted field distribution. An improved scheme for this correction method is discussed in Section 2.2.2.

The second type of resonances are intrinsic resonances, which occur if the spin tune is a linear combination of the betatron tunes. Hence, these resonances are driven by betatron motion. Certainly, the resonance strength can be reduced by aiming for small betatron oscillation amplitudes. Yet the more adequate method is to force a fast change of the vertical tune at the resonance specific time, such that the resonance crossing speed is increased. At ELSA, this method is successfully applied by using Panofsky type tune jump quadrupoles (for details see [23]).

During the SFB/TR16 the polarization in the external beam line of the CBELSA/TAPS experiment was increased to $(71 \pm 1) \%$ at $2.35 \mathrm{GeV}$ and $(62 \pm 2) \%$ at $2.92 \mathrm{GeV}$.

\subsubsection{Closed orbit correction update}

Reducing remaining horizontal magnetic fields plays a major role in polarization conservation. Vertical orbit displacements in quadrupole magnets will lead to horizontal magnetic field components, and hence the central goal is to keep especially the vertical orbit as flat as possible. Therefore, the beam should be steered through the magnetic centers of all quadrupole magnets. 


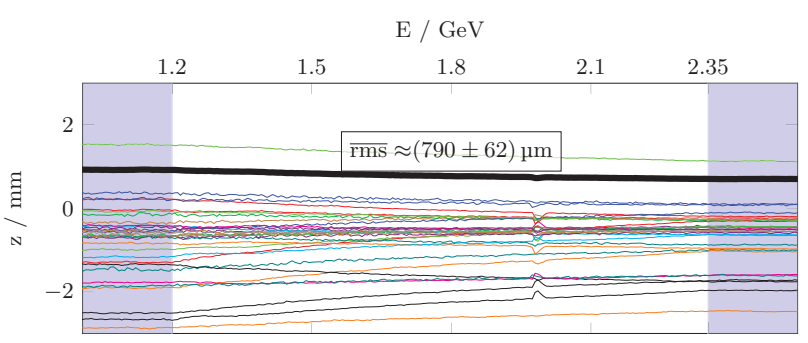

Figure 10. Development of the uncorrected vertical beam displacements at each of the 32 BPMs (bold black: $\overline{r m s}$ ). The energy is increased from $1.2 \mathrm{GeV}$ to $2.35 \mathrm{GeV}$ with $6 \mathrm{GeV} / \mathrm{s}$.

\section{Beam position monitors}

Each of ELSA's 32 quadrupole magnets is equipped with an in-house developed four-button monitor chamber (see Fig. 24 in Section 3.2). During booster mode operation the measurement of beam positions can be switched to a special readout mode: the internal readout rate is increased to $1 \mathrm{kHz}$ and position data is stored locally in the readout electronics of each beam position monitor (BPM). Up to 4095 data points can be stored, thus the development of beam displacements during the first four seconds (covering the injection, ramping and a large period of the extraction phase) of the ELSA cycle is available. Figure 10 shows the development of uncorrected beam positions at every quadrupole magnet during the fast energy ramp of $6 \mathrm{GeV} / \mathrm{s}$.

\section{Corrector magnets}

For horizontal correction the trim windings of the 24 dipole magnets are used. For correction in the vertical plane dedicated corrector dipole magnets had to be installed.

For the harmonic correction scheme (see Section 2.2.2) additional harmonic kick distributions varying sinusoidally a times along one revolution are applied every $440 \mathrm{MeV}$ during acceleration. Based on a precisely corrected vertical closed orbit (average rms value below $60 \mu \mathrm{m}$ during the energy ramp) vertical steerer magnet kicks are added. The vertical corrector system must be capable of changing corrector kicks between two consecutive integer spin tune resonances. At the maximum acceleration speed of $6 \mathrm{GeV} / \mathrm{s}$ this leads to a minimal time between two resonances of:

$$
\Delta t_{\mathrm{int}}=\frac{440 \mathrm{MeV}}{6 \mathrm{GeV} / \mathrm{s}} \approx 73 \mathrm{~ms} .
$$

In order to preserve the ability to perform orbit and harmonic corrections at energies up to $3.2 \mathrm{GeV}$ a new design for the vertical corrector magnets became necessary. In 2003, a new C-shape geometry for the magnet yokes was developed [17]. In the design phase two important parameters needed to be optimized: An integral corrector field strength $\left(\int B \mathrm{~d} s\right)$ greater than $9.6 \mathrm{mT} \mathrm{m}$ is needed. Additionally, the field rising time must not extend $70 \mathrm{~ms}$ to be capable to deal with the worst case situation where the
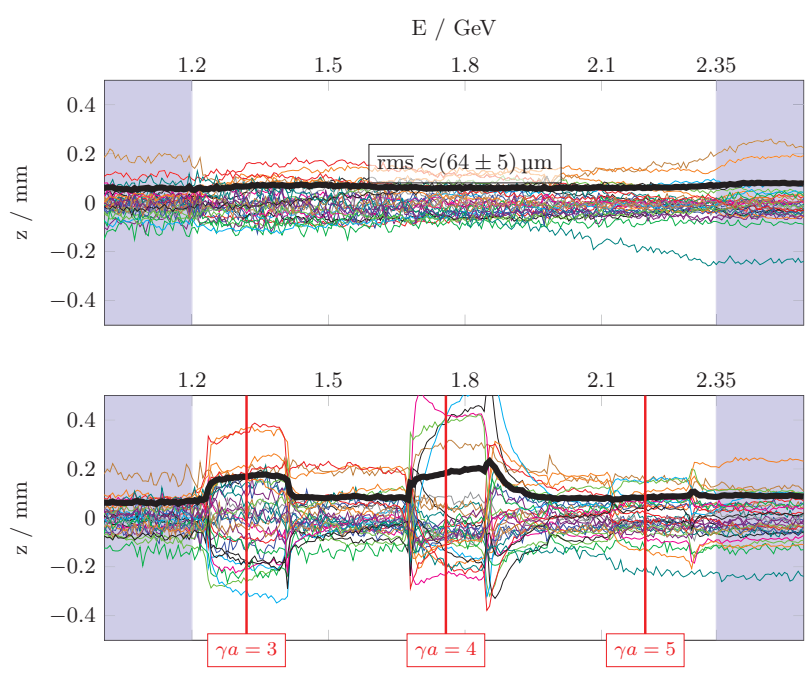

Figure 11. Development of the corrected vertical beam displacements at each of the 32 BPMs (bold black: $\overline{r m s}$ ). Top part: interpolated correction, lower part: additional harmonic corrections applied.

harmonic correction demands a complete field reversal between two integer resonances. Further consideration was given to field homogeneity and the fact that in several of ELSA's half cells the overall dimensions for installation of the magnets are quite narrow. The yokes are composed out of more than 300 thin metal sheets isolated against each other to prevent for eddy currents when fast field changes are requested. All 30 of these new magnets were installed during the last years.

Based on the measurements of the uncorrected beam positions (see Fig. 10) closed orbit corrections at the beginning and end of the energy ramp are calculated. According to these, linearly interpolated corrections are obtained and applied during the ramp. In the top part of Fig. 11 the development of the resulting corrected beam positions is shown. In the lower part additional kicks for harmonic correction of three integer resonances are applied.

\section{Power supplies}

Based on the requirements and a comprehensive analysis of commercially available power supplies it was decided to design and assemble new power supplies for all corrector magnets in-house. A key decision in the design phase was to use a pulsed full H-bridge consisting of 4 NMOStransistors. Together with a dedicated PI controller unit and a CAN bus driven communication device all these are placed in one 19 inch crate [43].

All required power supplies ( 24 powering the trim coils in the 24 bending dipoles for horizontal correction and 30 for vertical orbit correction) were installed in 2012 and have been in reliable operation since then.

\section{Closed orbit correction bandwidth}

In order to verify that the new orbit correction setup fulfills the desired requirements dedicated bandwidth studies 
were performed [20]. To determine the closed orbit correction bandwidth, which is the limiting bandwidth for resonance crossing, we measured the cutoff frequency of the whole system. This has been carried out by applying a sinusoidal excitation of a single steerer magnet and measuring the amplitude of the closed orbit distortion at that excitation frequency. The bandwidth of the system can then be defined as the frequency $f_{\text {cutoff }}$ at which the measured amplitude is reduced to $\frac{1}{\sqrt{2}}$. The correction bandwidth was determined to $(170 \pm 5) \mathrm{Hz}$.

High bandwidth closed orbit control is essential for acceleration of polarized electrons in a circular accelerator. The bandwidth measurements confirm that the closed orbit correction system fulfills the requirements and ensures the correction of all arising resonances even at the maximum acceleration speed.

\subsubsection{Improved correction scheme for integer resonances}

In the ELSA storage ring, integer resonances are compensated by destructive superposition of the depolarizing field component with a harmonic field distribution applied as kick angles with the orbit correction magnets (VC) around the ring:

$$
\alpha^{\mathrm{VC}}(\theta)=S \cdot \sin (\gamma a \cdot \theta)+C \cdot \cos (\gamma a \cdot \theta) .
$$

Here $\theta=\int_{0}^{s} 1 / R\left(s^{\prime}\right) \mathrm{d} s^{\prime} \in[0,2 \pi]$ is the spin phase advance changing only within the 24 bending magnets (finite curvature $1 / R$ ), where the spins precess around the vertical field. In between there are 24 segments $i$ of constant equidistant spin phase advance $\theta_{i}=i \cdot 2 \pi / 24$ with $i \in\{0,1, \ldots, 23\}$.

This correction procedure is called harmonic correction. The two parameters of the harmonic correction field $(S, C)$ have to be adjusted empirically for each resonance by iteratively optimizing the degree of polarization at the beginning of a beam time, because the resonance driving misalignments are not known precisely and may change over time. Figure 12 shows an example for such an optimization from March 2014. Here up to $25 \%$ polarization was gained from the harmonic correction of $\gamma a=3,4,5$.

\section{Previous correction scheme}

Naturally, the harmonic correction applied by the vertical correction magnets changes the vertical closed orbit. Thus, the horizontal fields acting on the beam in the quadrupoles also change. That is why the actual change of the resonance driving field distribution $\alpha^{\mathrm{H}}(\theta)$ by the harmonic correction not only consists of the set correction kicks $\alpha_{m}^{\mathrm{VC}}$ according to Eq. 1, but also implies quadrupole contributions $\alpha_{j}^{\mathrm{Q}}$ caused by the $\alpha_{m}^{\mathrm{VC}}$ :

$$
\alpha_{i}^{\mathrm{H}}:=\alpha^{\mathrm{H}}\left(\theta_{i}\right)=\sum_{m \in \mathrm{VC}} \delta_{m, i}^{\mathrm{VC}} \alpha_{m}^{\mathrm{VC}}+\sum_{j \in \mathrm{Q}} \delta_{j, i}^{\mathrm{Q}} \alpha_{j}^{\mathrm{Q}} .
$$

Here the abbreviations $\delta_{m, i}^{\mathrm{VC}}$ and $\delta_{j, i}^{\mathrm{Q}} \in\{0,1\}$ are used, which equal 1 if the corrector $m$ or quadrupole $j$ is located within

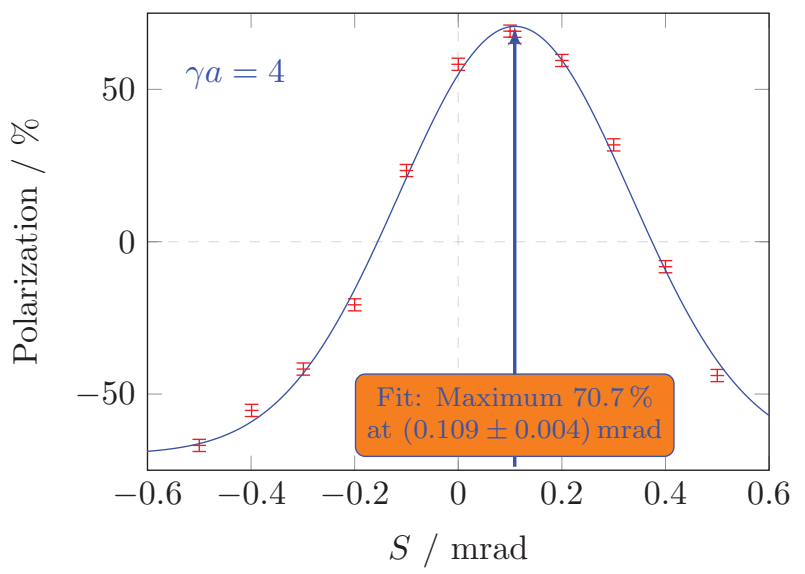

Figure 12. Møller polarimeter measurement from polarization optimization of integer resonance $\gamma a=4$ in March 2014. It took about $10 \mathrm{~min}$ to obtain one polarization value with a statistical error of $2 \%$. A Gaussian fit is used to determine the maximum [22].

the accelerator segment corresponding to the spin phase advance $\theta_{i}$.

Figure 13 demonstrates the impact of the previous correction scheme on the harmonic field distribution. For different set parameter pairs $(S, C)$ the change of the closed orbit $\Delta z$ was measured [22]. In each case the total kick distribution $\alpha^{\mathrm{H}}(\theta)$ was calculated and the actual measured parameter pair $(S, C)$ was determined from the corresponding frequency component via a Fast Fourier Transformation. Finally, each measured parameter is plotted against its corresponding set value. The plot shows no significant correlation between the set harmonic correction and the actual resulting field distribution. Although the resulting field changes with $(S, C)$, it is not the desired one. Nevertheless, the empirical polarization optimization is possible with this correction scheme (e.g. Fig. 12), but two problems may occur: First, a part of the parameter space may not be accessible at all, which could inhibit the optimization. Second, the consecutive optimization of both parameters may not result in the global maximum of polarization.

\section{Improved correction scheme}

An improved correction scheme was implemented [21] to achieve an accurate behavior of the harmonic correction. It does not apply the harmonic field distribution (Eq. 1) directly to the correction magnets, but considers the orbit response $\Delta z_{j}$ at the quadrupole $j$ to each correction magnet $m$ to determine the correction magnet kicks $\alpha_{m}^{\mathrm{VC}}$ needed for a proper sinusoidal harmonic distribution $\alpha^{\mathrm{H}}(\theta)$. Therefore, the quadrupole kicks are expressed via the orbit response matrix $O_{j m}=\partial z_{j} / \partial \alpha_{m}^{\mathrm{VC}}$, which is also used for the orbit correction, as $\alpha_{j}^{\mathrm{Q}}=l_{j} k_{j} \cdot \overrightarrow{O_{j}} \cdot \vec{\alpha}^{\mathrm{VC}}$. Now the so called harmonic correction matrix $\mathcal{H}$ can be defined to write the total kick caused by each correction magnet $m$ in each seg- 


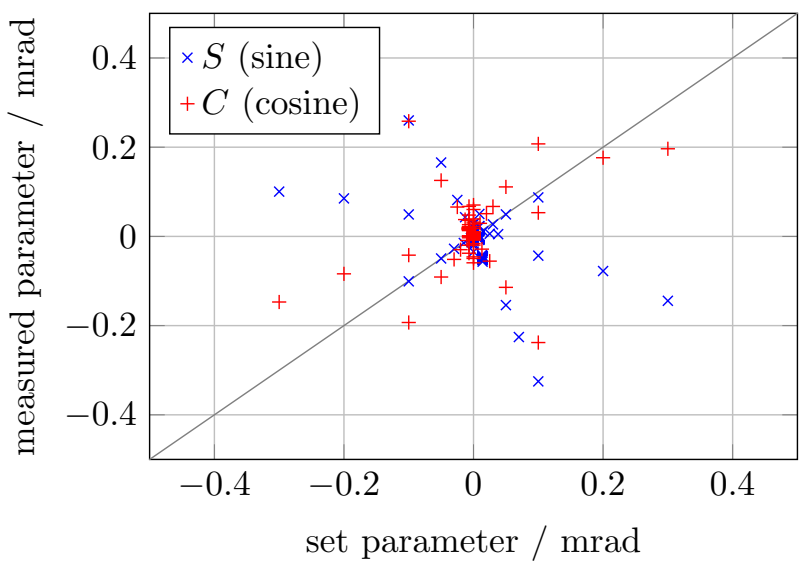

Figure 13. Parameters of the previous harmonic correction scheme for $\gamma a=3,4,5$. They deviate from the set parameters due to the quadrupole contributions caused by the orbit response to the correction fields [22].

ment $i$ as a matrix equation

$$
\mathcal{H}_{i m}:=\delta_{m, i}^{\mathrm{VC}}+\sum_{j \in \mathrm{Q}} \delta_{j, i}^{\mathrm{Q}} l_{j} k_{j} O_{j m}
$$

with quadrupole lengths $l_{j}$ and strengths $k_{j}$. Using this definition, Eq. 2 can be expressed as

$$
\vec{\alpha}^{\mathrm{HC}}=\mathcal{H} \vec{\alpha}^{\mathrm{VC}} \quad \Rightarrow \quad \vec{\alpha}^{\mathrm{VC}}=\mathcal{H}^{-1} \vec{\alpha}^{\mathrm{HC}} .
$$

$\mathcal{H}$ only depends on the orbit response and the accelerator lattice and optics. Finally, the pseudoinverse $\mathcal{H}^{-1}$ is determined numerically using a matrix inversion via single value decomposition.

Figure 14 shows the analysis of the actual parameters for the improved correction scheme [22] analog to Fig. 13. In contrast to the previous scheme, the harmonic field distribution is in good agreement with the set parameters. The improved correction scheme allows for accurate adjustment of harmonic field distributions in the accelerator, which is equivalent to direct control of individual resonances. For instance, it paves the way to a longitudinally polarized beam, which can be achieved if the beam is extracted exactly on an integer resonance and the precession is guided by the adjustable harmonic field distribution [44].

\subsection{Numerical simulation of the spin dynamics}

The final polarization at the experiment after the fast energy ramp in the ELSA storage ring depends on a large number of parameters including beam optics, magnet misalignments, individual setup of the energy ramp and all resonance correction schemes. Therefore, numerical simulations of spin dynamics are important for understanding and enhancement of operation with polarized beam. On the relevant timescale up to seconds, a common approach is tracking many individual 3D spin vectors through the fields of the accelerator and calculating polarization by averaging. Of course, this also implies particle tracking,

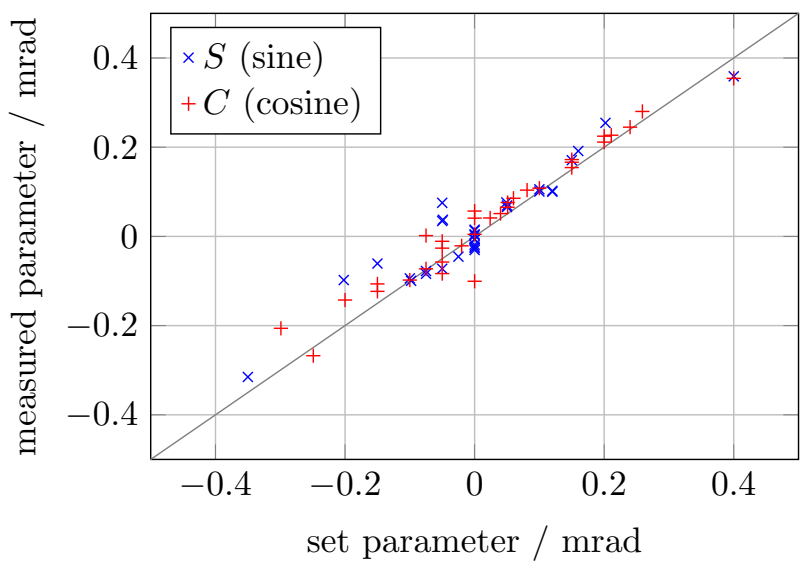

Figure 14. Parameters of the improved harmonic correction scheme for $\gamma a=3,4,5$. They are consistent with the set parameters, because the orbit response is included in the calculation of the correction fields [22].

because both synchrotron and betatron motion affect spin dynamics. Thereby, synchrotron radiation has to be taken into account, but many spin tracking codes do not cover synchrotron radiation, as they are commonly used for proton beams.

The new spin tracking code polematrix [27] was developed in-house at ELSA aiming to fulfill all requirements to model the energy ramp in the ELSA storage ring including a realistic synchrotron radiation model. polematrix solves the Thomas-BMT equation as

$$
\vec{S}\left(s_{0}+l\right) \approx \mathbf{R}_{\mathbf{3} \times \mathbf{3}}\left(s_{0}, l\right) \vec{S}\left(s_{0}\right),
$$

where the spin vector $\vec{S}$ is tracked from the position $s_{0}$ through a magnet with length $l$ by a three dimensional rotation matrix $\mathbf{R}_{\mathbf{3} \times \mathbf{3}}$. The matrix contains the spin precession in the magnet and is constructed from the corresponding energy normalized magnetic field $\overrightarrow{\widetilde{B}}:=e \vec{B} /(\beta \gamma m c)$. Its direction determines the precession axis and the rotation angle $\theta$ is calculated from the absolute value according to the Thomas-BMT equation:

$$
\theta=\vec{\xi} \cdot|\overrightarrow{\tilde{B}}(x, z)| \cdot l
$$

with transverse $\xi_{x}=\xi_{z}=\gamma a$ corresponding to the spin tune and longitudinal $\xi_{s}=a$. Here, $a$ is the gyromagnetic anomaly and $\gamma$ the Lorentz factor. The linear algebra calculations are performed using the $\mathrm{C}++$ library Armadillo[45]. Multiple spins can be tracked in parallel with an adjustable number of threads. Afterwards, the polarization vector is calculated as the average over all spin vectors for each output step. The program options are set in an xml configuration file.

Synchrotron motion and energy ramps are exclusively contained in $\gamma$ as a function of time for each particle. Transverse beam dynamics are included in the magnetic field $\overrightarrow{\widetilde{B}}(x, z)$, which depends on the horizontal $(x)$ and vertical $(z)$ particle trajectory. Several models for both longitudinal and transverse dynamics can be selected in the 
configuration file. One option is using the particle tracking from elegant as basis for the spin tracking. elegant is an established 6D particle tracking code, which includes synchrotron radiation and is actively developed at the Advanced Photon Source [46]. The particle trajectories are recorded during elegant execution and then imported by the spin tracking code.

The $C++$ particle accelerator lattice library libpalattice is used as interface to elegant. It is also developed at ELSA and was published as open source software [47]. libpalattice can be used to import any output of elegant or $M A D-X[48]$ into $C++$ programs. The tracking is executed automatically if needed. Basic parameters, like energy or number of turns, can be set directly in $C++$. Furthermore, libpalattice provides a convenient data structure to access and edit lattices with all element properties, which can also be automatically imported from and exported to elegant or $M A D-X$. So, a lattice file conversion tool is already included. Additionally, lattices can be exported to $\mathrm{ITT}_{\mathrm{E}} \mathrm{X}$ to sketch an accelerator with the tikz-palattice package [49].

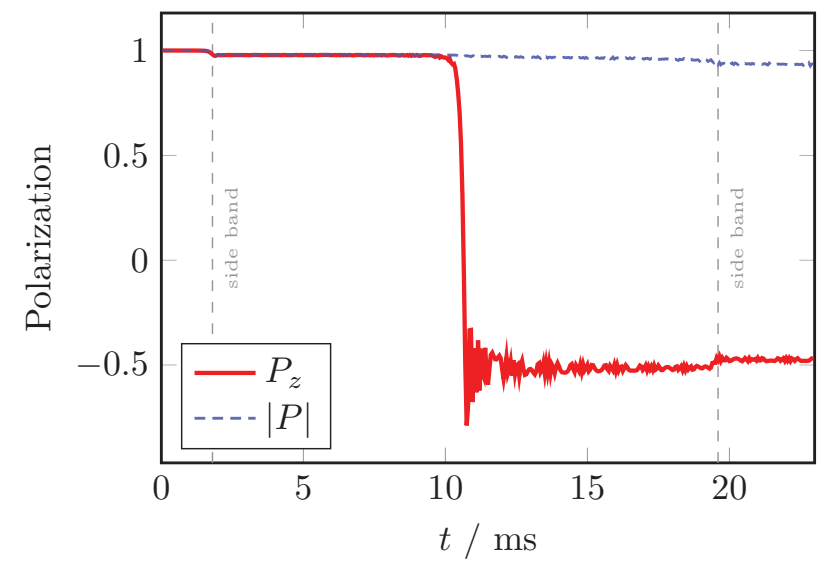

Figure 15. polematrix simulation of crossing an integer resonance with $4 \mathrm{GeV} / \mathrm{s}$ with 100 particles, spins starting in vertical direction.

Figure 15 shows a polematrix example of crossing the integer resonance $\gamma a=4$ with a linear energy ramp of $4 \mathrm{GeV} / \mathrm{s}$ in the ELSA storage ring. The vertical polarization $P_{z}$ is decreased by the resonance. Additionally, the individual synchrotron oscillations cause synchrotron side bands of the resonance. They reduce both $P_{z}$ and $|P|$, because the crossing speed of these sidebands is different for each particle resulting in incoherent spin motion. After the resonance, when the polarization vector is tilted out of the vertical direction $\left(P_{z}<|P|\right)$, synchrotron radiation leads to slow depolarization [27]. Combining many of these simulations allows to perform systematic studies of the resonance compensation measures. Figure 16 shows an exemplary 3D analysis of the harmonic correction discussed in Section 2.2.2. It visualizes the impact of the parameter correlation in the old correction scheme.

polematrix is a versatile tool for further optimizations to provide high degrees of polarization over the full energy range of ELSA. It will also be published as open source software soon.

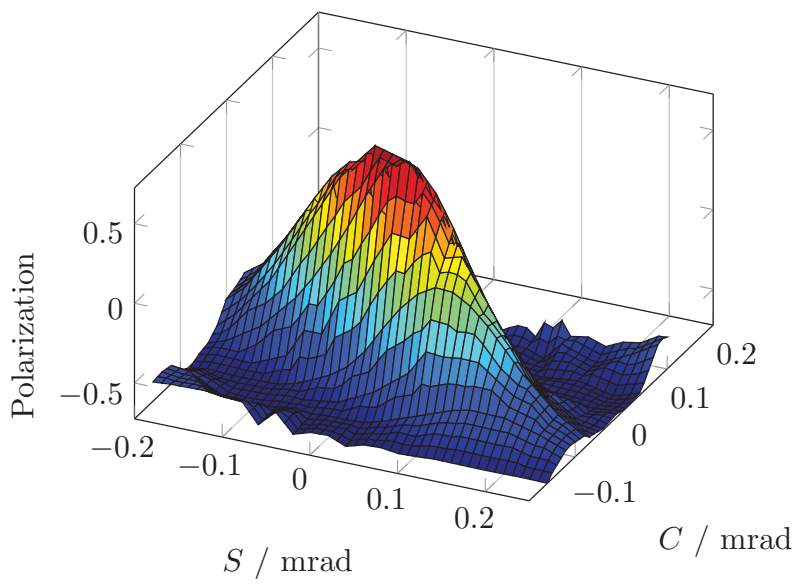

Figure 16. Simulation of the polarization after crossing $\gamma a=$ 6 depending on the setup of the previous harmonic correction scheme as discussed in Section 2.2.2.

\subsection{Compton polarimetry}

In addition to the Mott- and Møller polarimeters adjacent to the source and at the experimental stations, the electron polarization degree in the storage ring is observable via Compton scattering. As previous Compton polarimeter designs provided unsatisfactory measurement results due to insufficient laser power, pointing stability and detector noise, a revised polarimeter beamline was designed [28] and implemented.

\subsubsection{Polarization measurement technique}

A Yb:YAG disk laser provides two circularly polarized green laser beams which are brought to head-on collision with the transversely polarized electron beam (see Fig. 17). The intensity profile of the backscattered photons is detected with a silicon microstrip detector where the position of the profile's vertical centroid is a function of photon and electron polarization $P_{\gamma}$ and $P_{\mathrm{e}}$.

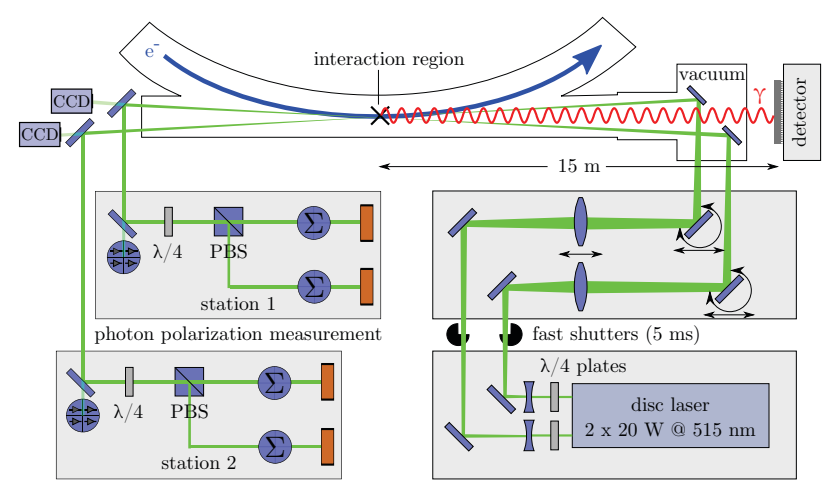

Figure 17. Layout of the Compton polarimeter. The microstrip detector measures the vertical profile of the backscattered photons. A laser beam polarization measurement station includes photo diodes for the measurement of the photon polarization [30]. 
When reversing $P_{\gamma}$ by the rotation of $\lambda / 4$ retarder plates, the profile centroid is displaced by

$$
\Delta z=\Delta z_{\max } \cdot P_{\gamma} P_{\mathrm{e}} .
$$

The electron polarization degree $P_{\mathrm{e}}$ is therefore obtained by measuring $P_{\gamma}$ and $\Delta z . \quad P_{\gamma}$ is evaluated behind the interaction region where the photon polarization is relinearized and characterized by a polarizing beam splitter (PBS) and position sensitive diode detectors (PSD). The analyzing power $\Delta z_{\max }$ is dependent on beam energy and its value is obtained by calibration utilizing the time-dependent self-polarization of an initially unpolarized, stored electron beam [50]:

$$
P_{e}=P_{\max } \cdot\left(1-\exp \left(-\frac{t}{\tau(E)}\right)\right),
$$

where the relaxation time $\tau$ is only dependent on the beam energy $E$ and $P_{\max }$ [51]. The maximum achievable polarization amounts to $P_{\max } \approx 92.4 \%$ if depolarizing effects are not considered, reducing the value otherwise.

Numerical simulations of the Compton-Process with the particle tracking toolkit Geant4[52] were carried out, taking into account the Gaussian nature of the laser beam, the dimensions of the silicon detector as well as ELSA's beam parameters. If the electron beam is extracted, ELSA's beam parameters differ from the case of stored electrons. The simulations showed, however, that the calibration factor $\Delta z_{\max }$ will not be altered during extraction.

The intensity profile of backscattered photons on the detector is overlapped by beam gas radiation with similar photon energy. This background is evaluated and subtracted in a separate measurement with blocked laser beams. Signal as well as background are proportional to the electron current.

\subsubsection{Detector system}

A silicon microstrip detector in combination with a lead conversion target of two radiation lengths was chosen for measuring the backscattered photons [30]. The silicon sensor is AC-coupled and has a thickness of $300 \mu \mathrm{m}$. It covers a surface of approximately $4 \mathrm{~cm} \times 4 \mathrm{~cm}$ with 768 horizontally orientated strips of $50 \mu \mathrm{m}$ pitch. With an expected width of the intensity profile of backscattered photons of about $1 \mathrm{~cm}$ and $\Delta z_{\max }=70 \mu \mathrm{m}$, a polarization measurement error of less than $1 \%$ is expected.

The detector readout electronics is FPGA based and allows the processing of the expected photon rate of about $1 \mathrm{MHz}$. For this purpose, dedicated ASIC chips were developed in close cooperation with the SiLab/ATLAS group of the University of Bonn. The charge threshold for which a hit is counted was set to 3500 electrons (with a minimum ionizing particle producing about 23000 electrons in $300 \mu \mathrm{m}$ silicon). This results in a noise rate of less than 1 count $/ \mathrm{min}$

\subsubsection{First polarization measurements}

The performance of the detector system and optical beamline was tested during several measurements with a self-

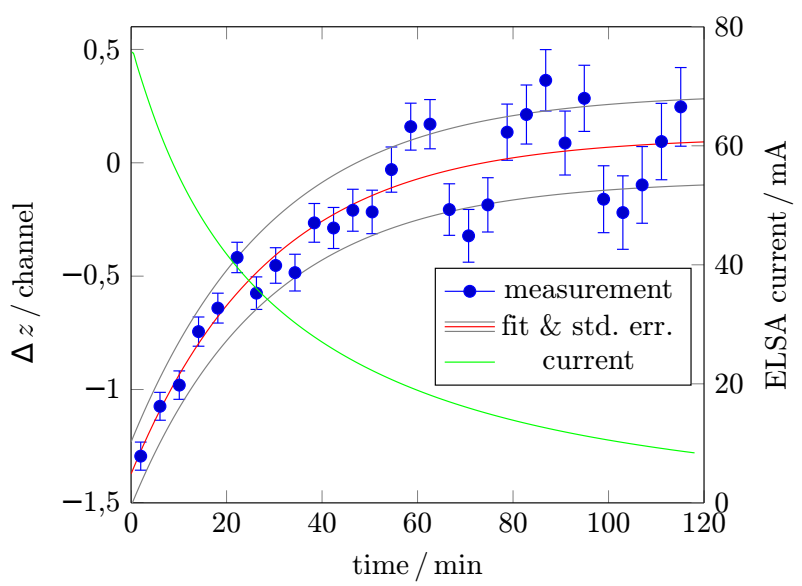

Figure 18. Vertical shift $\Delta z$ of the backscattered photons due to self-polarization of the stored electron beam. The SokolovTernov effect is utilized to calibrate the analyzing power of the Compton polarimeter.

polarizing stored electron beam. Fig. 18 shows the development of $\Delta z$ as a function of time. Initially, a current of $75 \mathrm{~mA}$ at $2.35 \mathrm{GeV}$ was injected into the storage ring. The available laser power varied between $5 \mathrm{~W}$ to $12 \mathrm{~W}$ and a circular polarization degree of about $98.6 \%$ was estimated. The data fit according to Eq. 3 determines the relaxation time $\tau=(28.9 \pm 6.4) \mathrm{min}$ in good agreement with the expected value from theory, neglecting depolarizing effects. Due to the decreasing stored current the measurement error (which is purely statistical) is yet too large to allow conclusions on the strength of depolarizing effects which influence the value of $P_{\text {max }}$. Higher laser power for a better signal to background ratio would therefore be necessary. The displacement offset results from a reproducible angular shift of the laser beam when turning the quarter-wave plates, which was confirmed by the installed laser beam diagnostics.

\section{High beam intensities}

One major part of the ELSA upgrade program consisted in an increase of the external beam current by one order of magnitude to a maximum value of about $10 \mathrm{nA}$. When keeping the macroscopic duty cycle of the post accelerating mode at the desired values of about $80 \%$, the envisaged intensity upgrade can only be achieved by increasing the current in the storage ring to a maximum value of about $200 \mathrm{~mA}$. To supply the required beam current, the existing injector LINAC 1 has been overhauled, which included the redesign and construction of several major components [53-56].

Different measures have been undertaken to suppress the excitation of beam instabilities in the storage ring. The overall impedance was reduced significantly by replacing the majority of the beam pipes by newly developed ones with smooth geometry. A simple higher order modes (HOM) damping system and a new temperature stabilization have been developed and installed at the accelerat- 
ing PETRA cavities resulting in a significant reduction of HOM driven beam instabilities [57]. To achieve effective damping of the remaining excitation of multi-bunch instabilities, a powerful bunch-by-bunch feedback system has been developed and successfully set up at the storage ring [58-63]. In addition, a precise stabilization of the rf amplitude and phase, as required by the new bunch-by-bunch feedback system, has been achieved by installation of a new low level rf system (LLRF) which is based on digital signal processing [64].

Using the diagnostic capabilities of the feedback system, a fast measurement and stabilization procedure of the transverse tunes has been developed and set up. Monitoring transverse and longitudinal beam dynamics with temporal resolution of picoseconds were made possible with the installation of a streak camera system at the storage ring [65-69].

\subsection{High current and single bunch injector}

The new high current injector is based on the existing normal conducting S-band traveling wave accelerating structure of LINAC 1. It has been equipped with a new pulsed triode thermionic gun, a prebuncher cavity operating at a subharmonic frequency of $500 \mathrm{MHz}$ and a 4-cell S-band traveling wave buncher (TWB) which all were taken from the S-band test facility (SBTF) at DESY. Major parts of the rf power system of the linac and the transfer line to the booster synchrotron were completely rebuilt, and a new energy compression system has been designed and constructed. The final commissioning of the new injector will start right after finishing the installation of the new components. In Fig. 21 an overview of the complete setup is presented.

\subsubsection{Gun and bunching system}

The $90 \mathrm{kV}$ electron source uses the original components of the thermionic gun of the SBTF at DESY. According to the considerably lower emission current required for the operation at ELSA, the cathode conus has been redesigned and rebuilt. For this purpose, the emission characteristics of the gun have been optimized by simulating the beam propagation with the software EGUN[32] to enable a single pulse operation with $1.5 \mathrm{~A}$ beam current as well as long pulse operation with $0.8 \mathrm{~A}$ [53]. Further bunching is achieved by the prebuncher and the TWB. To prevent phase shifts, all $\mathrm{rf}$ cavities and structures are powered by one $500 \mathrm{MHz} 2 \mathrm{~W}$ continuous wave rf generator, whose frequency is sextupled before feeding the klystrons that power the S-band structures. Measurements of the gun's and prebuncher's performance in the single bunch mode have been carried out using the wall current monitors installed at different positions in the transfer line to the linac (WCM1 - WCM3) [55] and are presented in Fig. 19. The measured pulse lengths $(2 \sigma)$ amount to $(1.02 \pm 0.04) \mathrm{ns}$ behind the electron gun, $(1.08 \pm 0.04) \mathrm{ns}$ before the prebuncher and $(0.41 \pm 0.04) \mathrm{ns}$ at the linac entrance, demonstrating that the requirements for a successful single bunch accumulation can be fulfilled.

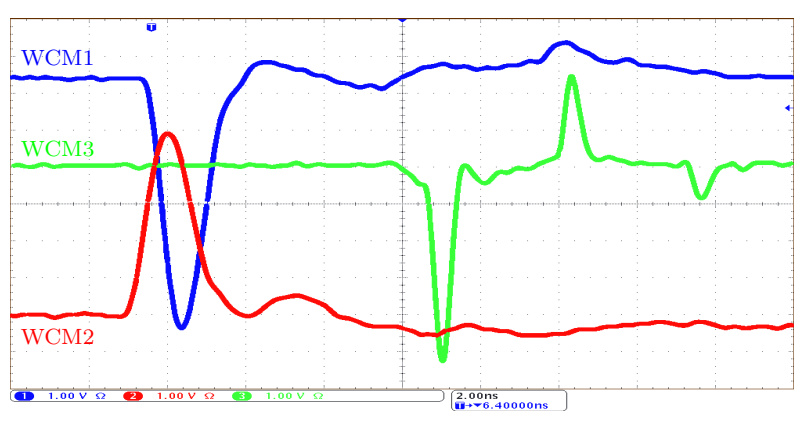

Figure 19. Longitudinal intensity profiles measured using the wall current monitors (WCM) installed in the transfer line at the linac. The absolute signal positions depend on the cable lengths, the signal polarities depend only on the way the connector is linked to the WCMs [55].

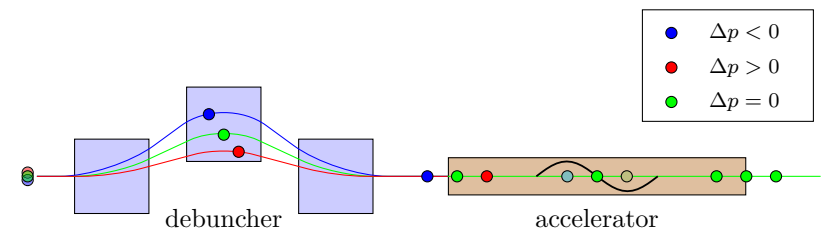

Figure 20. Scheme of the energy compression system [70].

\subsubsection{Energy compression system}

The redesigned transfer line to the booster synchrotron has been constructed and commissioned using already existing magnets (dipole bends and focussing quadrupoles). The total number and the positions of the individual quadrupole magnets have been changed according to detailed simulations of the optics, which were carried out using the software package $M A D-X$. In order to enhance the injection efficiency into the booster synchrotron which is limited by its energy acceptance of $0.5 \%$, the energy spread of the electron beam of LINAC 1 has to be reduced by one order of magnitude. Despite using the multi-stage bunching process of the injector, the energy spread has to be further reduced after acceleration in the main linac. This is achieved by adding an energy compression system.

A dispersive debuncher section is used to rotate the phase space distribution of the electron beam in order to sort the electrons by their momentum, using three dipole magnets. Different momenta lead to different curvatures and thus to different path lengths. The resulting longitudinal particle distribution is sorted by the particle's momentum. The adjacent accelerating structure is used to apply a longitudinally alternating electric field to the debunched beam in order to decelerate or re-accelerate electrons depending on their longitudinal position in the bunch. By choosing an appropriate if phase for this acceleration, the energy spread can be reduced by about one order of magnitude. A scheme of the energy compression is given in Fig. 20.

The accelerating structure of the energy compression system is a normal conducting, 28 cell $3 \mathrm{GHz}$ rf travel- 


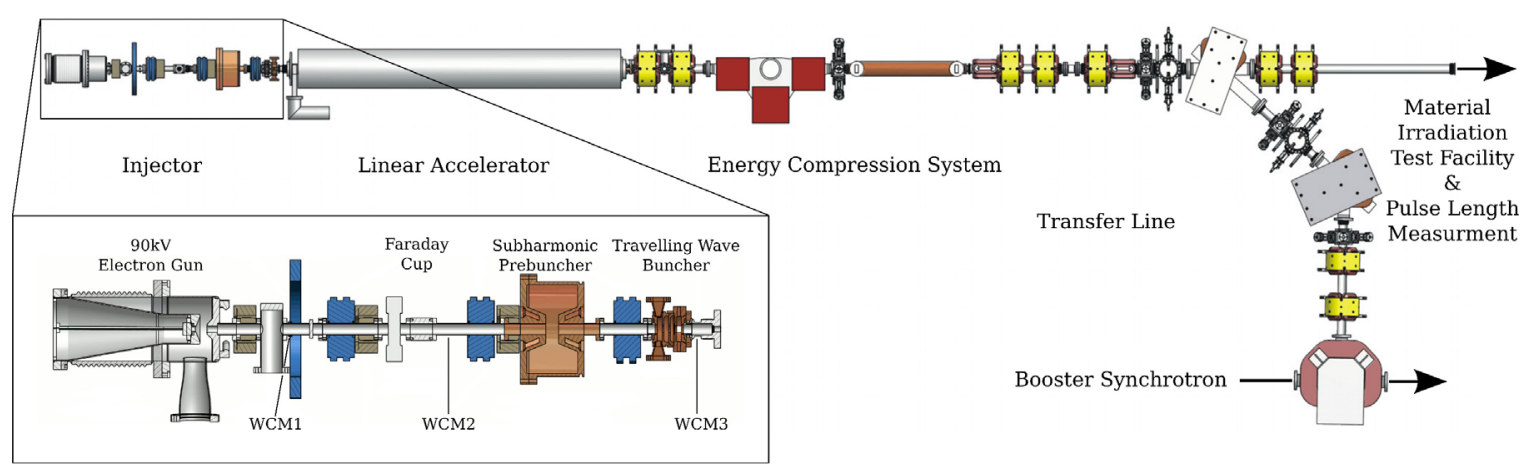

Figure 21. The new linear accelerator facility at ELSA [55].

ing wave structure, manufactured by Research Instruments $\mathrm{GmbH}$.

The output power of the main klystron is split to simultaneously drive traveling wave buncher, main linac and energy compression structure. The rf amplitude and phase of both, traveling wave buncher and energy compression system, can be changed relatively to the main linac using a hybrid based high power in-vacuum waveguide system to achieve a maximum bunching and energy compression effect.

\subsubsection{Hybrid based waveguide system}

To drive the traveling wave buncher, the main linac and the energy compression accelerating structure, a pulsed $3 \mathrm{GHz}$ klystron is used. The rf power is distributed to the three accelerating structures with individual and adjustable rf amplitudes and phases using a high power WR284 waveguide system based on $3 \mathrm{~dB}$ hybrids and phase shifters. Figure 22 shows the schematics of the new waveguide system.

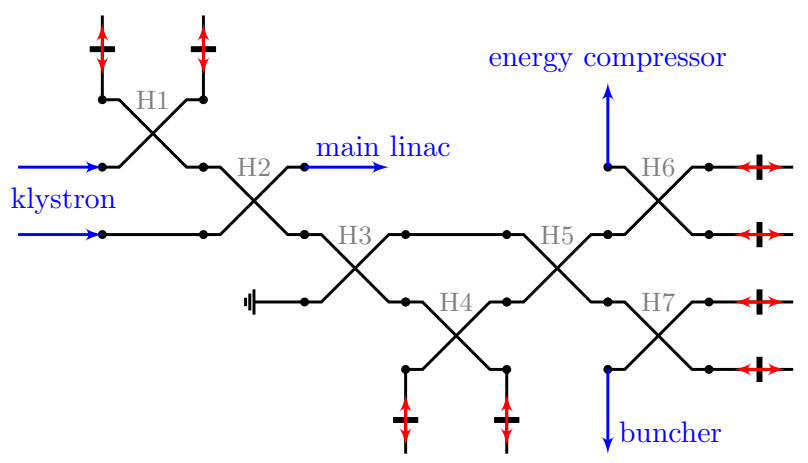

Figure 22. Schematics of the new waveguide system including $3 \mathrm{~dB}$ couplers and variable waveguide shorts [56].

The two output arms of the main klystron are combined using the hybrid coupler H2. To couple the main part of the rf power to the main linac and a small part to the bunching and energy compression structures, the hybrid $\mathrm{H} 1$ is used as a phase shifter by shorting its two output ports, using position variable in-vacuum rf shorts shown in Fig. 23. By varying the position of the shorts synchronously only the output phase of the hybrid with respect to the input one is affected. The waveguide shorts are based on a design of TU Dortmund [71] and have been modified to fulfill ultra high vacuum requirements.

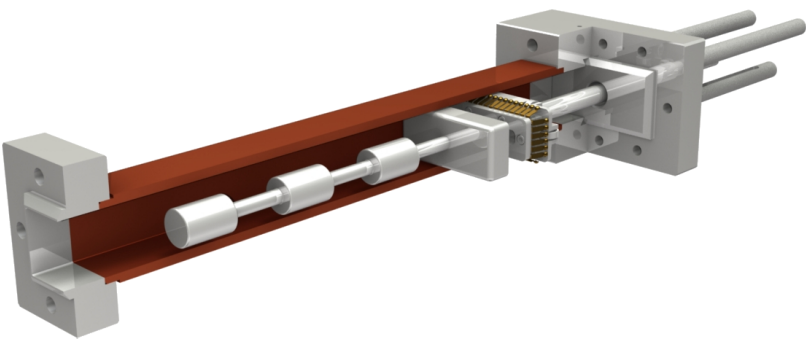

Figure 23. Variable in-vacuum high power waveguide short [56].

In the same way, a hybrid can be used as a splitter by driving a single input port, which is used in H3. To avoid damages from undesired reflected powers, the second input port of $\mathrm{H} 3$ has to be terminated using a water cooled rf load.

These schemes are used in hybrids $\mathrm{H} 4$ and $\mathrm{H} 5$ as well, splitting the remaining rf power for traveling wave buncher and energy compression structure. The adjacent hybrids $\mathrm{H} 6$ and $\mathrm{H} 7$ are connected to the energy compression structure and the traveling wave buncher and are used as phase shifters for both paths individually.

The complete system has been designed to handle powers of up to $30 \mathrm{MW}$ and allows full amplitude and phase control of the three output paths by varying the position of the waveguide shorts.

\subsection{Low impedance vacuum chambers}

In case of sudden changes in the geometry of vacuum chambers, strong wakefields may be excited. Depending on the type of the geometry mismatch, the resulting broadband or narrowband impedances will cause single or multi-bunch instabilities. In order to significantly reduce the ELSA vacuum chambers' total impedance, new elliptical chambers for major parts of the straight sections in the storage ring have been developed and constructed. The new chambers in the quadrupole magnets (see Fig. 24) have been equipped with capacitive pick-up beam position 


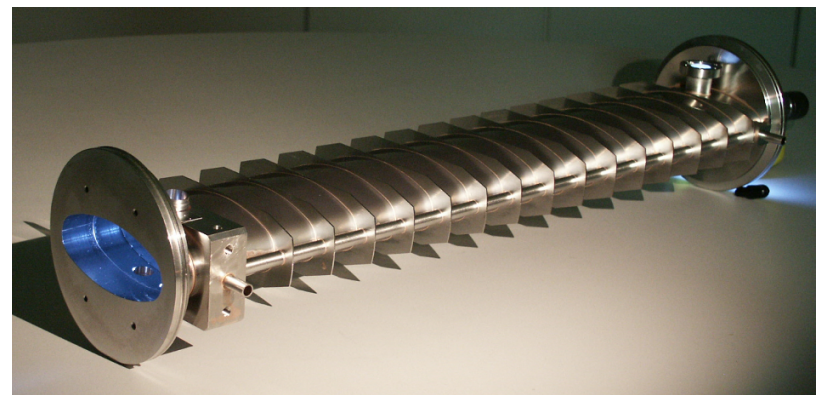

Figure 24. Newly developed water-cooled quadrupole chamber with capacitive pick-up beam position monitor (left end) and ion clearing electrode (right end).

monitors (BPM) and clearing electrodes with a bias voltage of up to $3 \mathrm{kV}$ to efficiently remove ions captured by the electron beam.

Almost all pumping crosses were replaced by newly constructed ones, where special effort has been made to maintain a smooth beam pipe geometry (see Fig. 25).

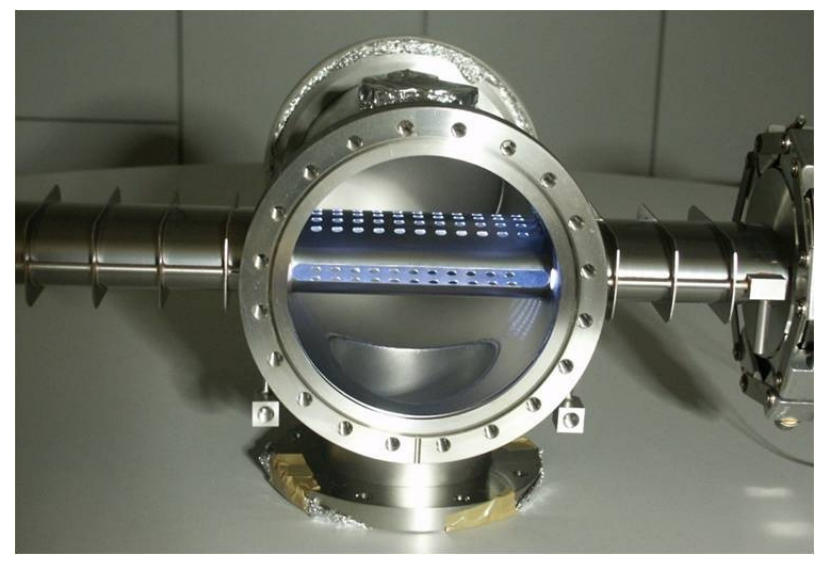

Figure 25. Newly developed water-cooled pumping cross with smooth beam pipe geometry.

In addition, the interconnections of the curved dipole and straight quadrupole chambers have been modified to reduce their impedance. Spiral springs have been inserted to ensure electrical contact. The existing ceramic interfaces at both ends of the bended dipole chambers were bridged by dedicated clips made from copper beryllium (see Fig. 26).

With these modifications, the total impedance of the vacuum chambers has been reduced significantly. Nevertheless, it is assumed that still a considerably high impedance is present which is mainly caused by an inadequate construction of vacuum components like kicker tanks and septum magnets.

\subsection{Higher order modes}

Higher order modes (HOM) of the accelerating cavities are one of the dominant sources of multi-bunch instabilities in storage rings. If the eigenfrequency of a specific

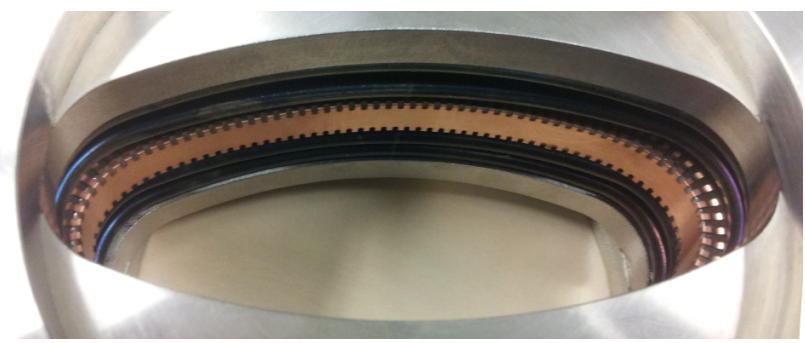

Figure 26. Mounted rf clip made from copper beryllium to bridge the ceramic break at the ends of the bended dipole vacuum chamber [70].

HOM overlaps with one of the beam's synchrotron sidebands, this HOM will be excited and may lead to strong beam instabilities which deteriorate the beam quality and can cause severe beam loss when increasing the beam current. Beside an effective damping of all HOMs excited by the circulating beam, a shift of the most harmful HOMs to frequencies apart from the synchrotron sidebands proves to be an effective way of HOM suppression. Both measures have been successfully deployed at ELSA.

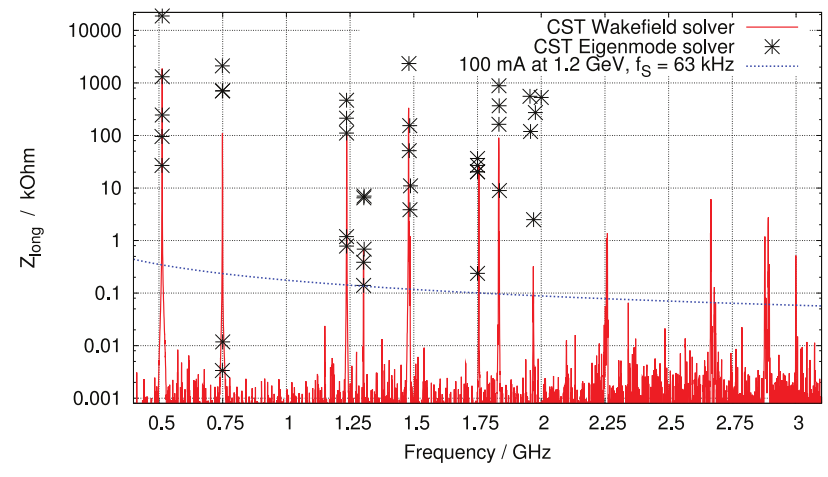

Figure 27. Wake impedance and natural damping calculations of a five-cell PETRA cavity using CST Microwave Studio ${ }^{\circledR}$ [63].

\subsubsection{HOM spectrum}

The most important measure for the perturbing effect of a specific HOM on the beam is its longitudinal impedance. To estimate the HOM impedances of a PETRA cavity, its geometry was modeled within CST Microwave Studio ${ }^{\circledR}[72]$. Using the $C S T^{\circledR}$ wakefield and eigenmode solver, the impedances of all PETRA HOMs up to $3 \mathrm{GHz}$ have been calculated. The resulting spectrum including the natural damping threshold is shown in Fig. 27 which has been calculated for a circulating beam current of $100 \mathrm{~mA}$. Besides the fundamental mode at $500 \mathrm{MHz}$, which is used for particle acceleration, the $\mathrm{TM}_{021}$ mode at $1.46 \mathrm{GHz}$ has an estimated impedance well above $1 \mathrm{M} \Omega$. In addition, many other HOMs show up with impedances exceeding $100 \mathrm{k} \Omega$. A measurement of the impedance of all relevant HOMs has been performed applying the bead-pull technique [73] at a structurally identical spare PETRA cavity. For the $\mathrm{TM}_{021}$ mode an impedance of 


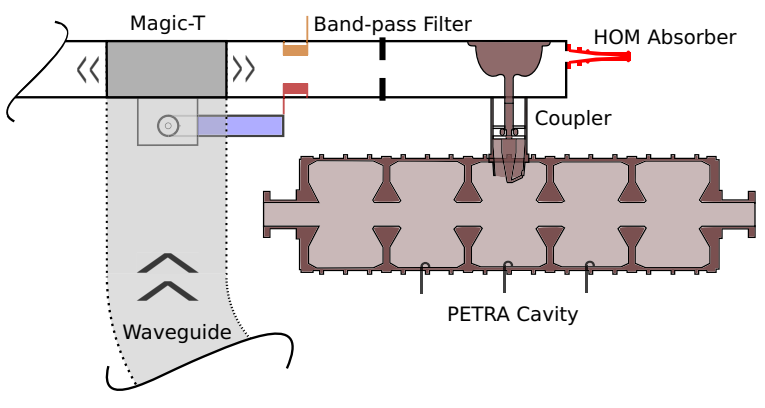

Figure 28. Schematic excerpt of the waveguide system with HOM absorbers attached to the PETRA cavity.

$(1.221 \pm 0.191) \mathrm{M} \Omega$ was measured, confirming the results of the $C S T^{\circledR}$ simulations. This indicates that in particular this mode can cause multi-bunch instabilities.

\subsubsection{HOM damping in the waveguide system}

If a HOM is excited by the electron beam, it can partially propagate into the attached waveguide system, which directs the rf power from the klystron to the cavities. To reduce the impedance of all HOMs, broadband HOM absorbers in combination with $500 \mathrm{MHz}$ band-pass filters were installed at the waveguide system (see Fig. 28). Whereas the $500 \mathrm{MHz}$ rf power from the klystron is transmitted through the band-pass filter, HOMs which propagate inside the waveguide system get reflected back and are attenuated by the HOM absorbers. Due to the absorber's cut-off frequency of about $900 \mathrm{MHz}$, the $500 \mathrm{MHz}$ rf power is not affected and completely transmitted into the cavity. Thus the absorber provides additional damping for all HOMs which are subject to coupling to the waveguide system via the fundamental input coupler.

\subsubsection{Temperature stabilization and HOM shifting}

For beam currents up to $100 \mathrm{~mA}$ mainly longitudinal multi-bunch instabilities are excited at ELSA. As expected, especially the $\mathrm{TM}_{021}$ mode at $1.46 \mathrm{GHz}$ is causing longitudinal beam instabilities by driving the lower synchrotron sideband of the 21 st revolution harmonic. With the harmonic number being 274, this corresponds to an excitation of the multi-bunch mode 252 which showed up as the only and dominating mode in the recorded spectrum of multi-bunch modes [63]. Particular attention was therefore drawn to shift the frequency of this most harmful mode. This can be achieved by rising the operating temperature of the cavities. Since the resonance frequencies of all eigenmodes are defined by the cavity's geometry, a thermal expansion shifts all HOMs to lower frequencies. Whereas the fundamental mode is fully restored by the tuner loop control, a residual frequency shift of the $\mathrm{TM}_{021}$ mode remains.

An increase of the cavities' operating temperature can easily be achieved by reducing the flow rate of the cooling water. To maintain full temperature control, an isolated cooling circuit was integrated for each cavity (see

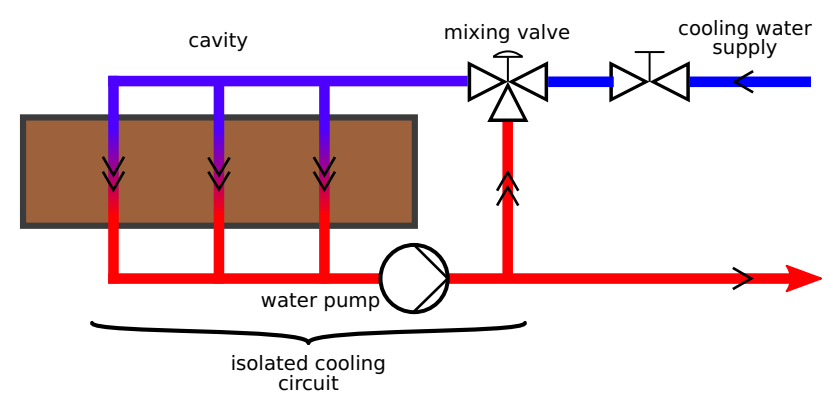

Figure 29. Schematic of the temperature stabilization of the PETRA cavities.

Fig. 29). The body temperature of each cavity is controlled by a mixing valve adjusting the admixture of cool water from the main cooling system into the local circuit. The temperature is stabilized using PID controllers.

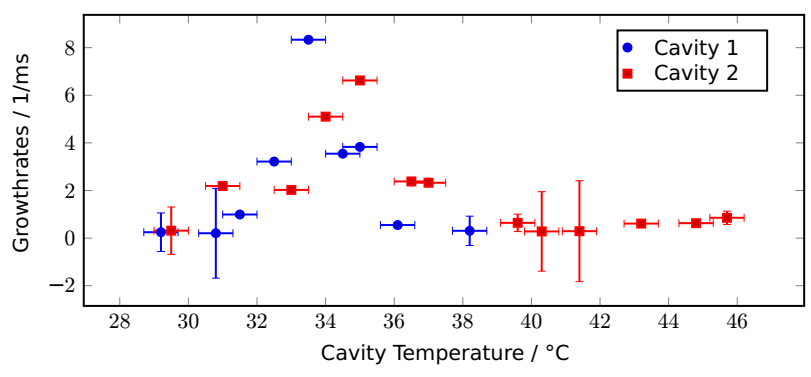

Figure 30. Growth rate of the multi-bunch mode 252, depending on the temperature of the PETRA-Cavities at a beam current of about $35 \mathrm{~mA}$ [70].

Figure 30 shows the measured growth rate of the multibunch mode 252 , which is excited by the $\mathrm{TM}_{021}$ mode of the PETRA cavities. The measurement confirms the strong dependence on the cavity temperature. Rising the cavities' temperature to $>38^{\circ} \mathrm{C}$ leads to a remarkable decrease of growth rate, which allows a stable storage of significantly higher beam currents.

\subsection{Bunch-by-bunch feedback system}

To damp the multi-bunch oscillations excited in the longitudinal and transverse planes, a state-of-the-art bunchby-bunch feedback system has been successfully set up at the ELSA storage ring. The system consists of four major parts: A beam position monitor (BPM) picks up a signal corresponding to the bunch's position. Then, the frontend of the feedback system mixes the BPM signal with the third harmonic of the ELSA $500 \mathrm{MHz}$ master rf to isolate a signal only including information about the beam oscillations. By amplitude or phase demodulation the requested properties can be obtained: Using phase demodulation, the longitudinal position of every single bunch can be extracted. Amplitude demodulation allows for measuring the transverse position. A fast ADC converts the signals into digital format. Using a field programmable gate array (FPGA), a digital filter with a maximum of 


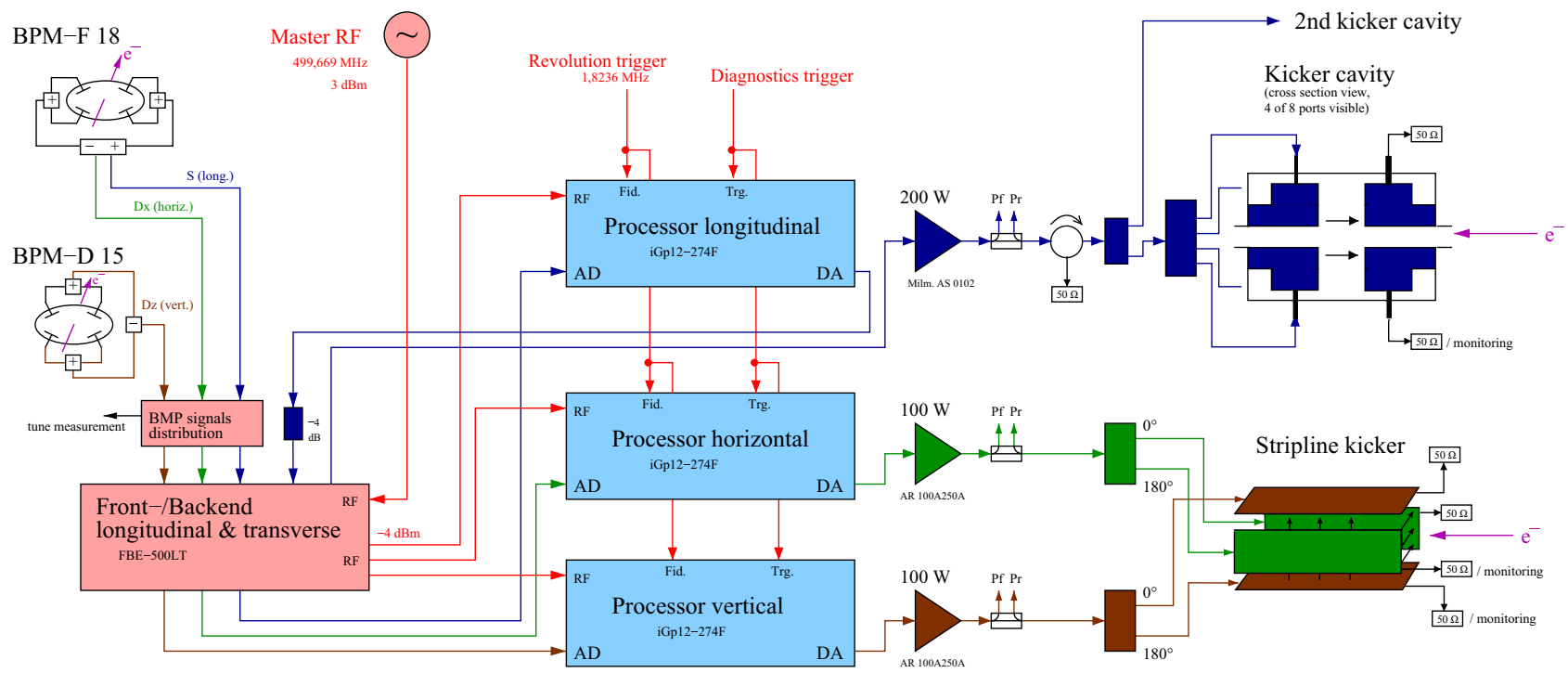

Figure 31. Schematic overview of the longitudinal and both transversal bunch-by-bunch FB systems with all main components installed at the ELSA storage ring [58].

32 taps isolates the oscillation frequency of every single bunch, allowing to generate a correction signal which is sent to a fast DAC. The resulting correction signal requires broadband processing, reaching from DC to a maximum of $250 \mathrm{MHz}$, which is half of the accelerating rf. In the transverse planes, two broadband rf amplifiers in use ensure a sufficient $\mathrm{rf}$ power for a proper damping of every single bunch by the connected stripline kicker. In the longitudinal plane, the attached kicker cavity operates at $1 \mathrm{GHz}$ to $1.25 \mathrm{GHz}$, which requires a mixing of the correction signal with the second rf harmonic before amplification. An overview of the feedback system including all major components is given in Fig. 31. The digital signal processing units (DSP) have been purchased as commercially available modules of Dimtel Inc. [74]. Both stripline kicker and kicker cavity are in-house developments adjusted to the ELSA rf frequency and bunch length.

\subsubsection{Longitudinal kicker cavities}

Due to the frequency range of all multi-bunch oscillations, the center frequency $f_{\mathrm{c}}$ of a kicker cavity for the longitudinal feedback system should be configured to

$$
f_{\mathrm{c}}=\left(N \pm \frac{1}{4}\right) f_{\mathrm{rf}}, \quad N \in \mathbb{N}
$$

Considering the quite large bunch length of up to $6 \mathrm{~cm}$ at an energy of $3.2 \mathrm{GeV}$ in the storage ring, we have chosen $N=2$ which corresponds to a kicker cavity center frequency of $1.125 \mathrm{GHz}$ and an operating range of $1.0 \mathrm{GHz}$ to $1.25 \mathrm{GHz}$. The kicker cavity is designed as a waveguide loaded pillbox cavity with a cylindrically symmetrical beam pipe. The geometry of the cavity is based mainly upon several developments at other accelerator facilities [75-77]. The large bandwidth of $250 \mathrm{MHz}$ required to damp all multi-bunch modes is obtained by loading the fundamental mode of the cavity with four single-ridged waveguides on each cavity side which leads to a significantly small cavity's quality factor.

Furthermore, eight broadband waveguide-to-coaxial transitions and rf feedthroughs provide the external connection via type $\mathrm{N}$ connectors. Four of them are used for the input signals driving the cavity, the other four are terminated by water-cooled $50 \Omega$ loads or are usable for monitoring. Figure 32 shows a picture of the final installation of both in-house fabricated kicker cavities.

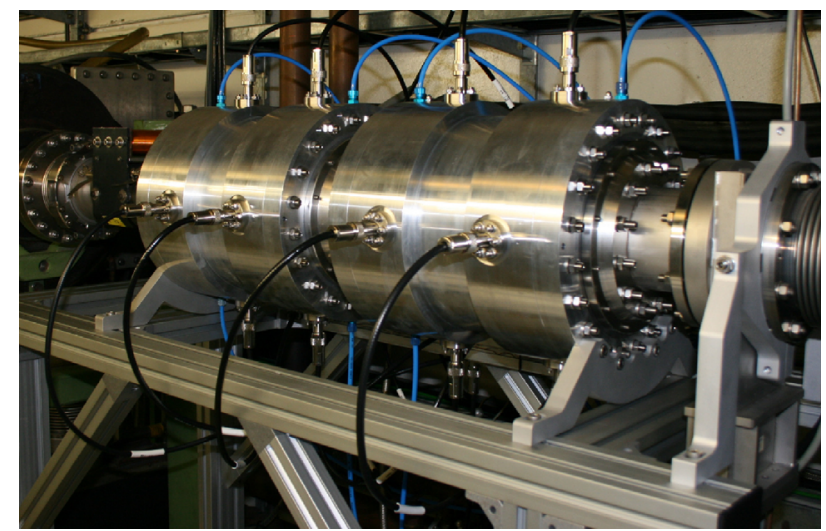

Figure 32. Longitudinal kicker cavities installed at the ELSA storage ring [62].

The geometry of the cavity was determined from numerical simulations carried out with CST Microwave Studio $^{\circledR}\left(M W S^{\circledR}\right)$. For details of the geometry and the design parameters we refer to [60]. The cavities' scalar reflection coefficient $\mathrm{S}_{11}$ simulated by using the $M W S^{\circledR}$ Transient Solver is shown in Fig. 33 and is characterized by a large bandwidth of $\Delta f=254 \mathrm{MHz}$ and an almost zero reflection (critical rf coupling) at the center frequency of $1.102 \mathrm{GHz}$. 
Table 1. Selected kicker cavities' design parameters, simulation and measurement results.

\begin{tabular}{lcccccc}
\hline Parameter & Specified & Simulated & \multicolumn{2}{c}{ Measured cavity 1 } & \multicolumn{2}{c}{ Measured cavity 2 } \\
\hline center frequency $f_{\mathrm{c}} / \mathrm{MHz}$ & 1125.0 & 1101.0 & 1125 & \pm 1 & 1126 & \pm 1 \\
bandwidth $\Delta f / \mathrm{MHz}$ & 250.0 & 254.0 & 297 & \pm 32 & 339 & \pm 18 \\
shunt impedance $R_{\mathrm{S}} / \Omega$ & & 414.0 & 338 & \pm 15 & 316 & \pm 12 \\
quality factor $Q$ & 4.5 & 4.3 & 3.8 & \pm 0.1 & 3.3 & \pm 0.1 \\
\hline
\end{tabular}

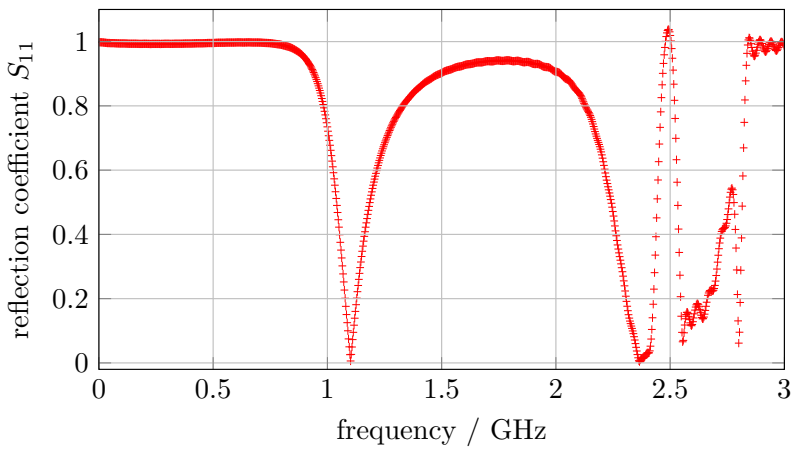

Figure 33. Scalar reflection coefficient of the kicker cavity as a function of frequency until $3 \mathrm{GHz}$ simulated by $M W S^{\circledR}$ Transient Solver. The broadband fundamental mode at $1.1 \mathrm{GHz}$ is clearly visible. First HOMs do not occur below $2.3 \mathrm{GHz}$ and will be damped due to their propagation through the beam pipe [62].

The cavities' geometry was adapted to this frequency, which is lower than the specified value of $1.125 \mathrm{GHz}$. This is due to compensation for a frequency shift between the simulation results and the measurement of the resonance curve of a prototype cavity fabricated during the design phase. Thereby, both later fabricated and installed kicker cavities have a center frequency of nearly $1.125 \mathrm{GHz}$ which is confirmed by the measured resonance curve of both cavities presented in Fig. 34. The corre-

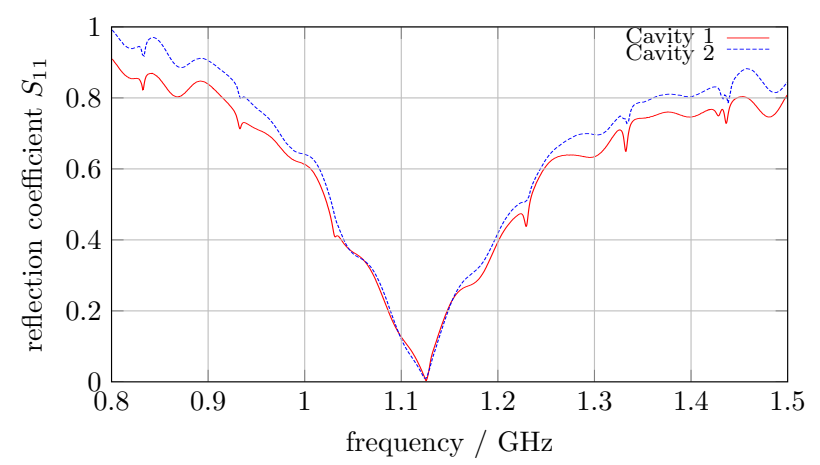

Figure 34. Measured resonance curves of both installed kicker cavities.

sponding fundamental mode of the cavity excited at the center frequency is the $\mathrm{TM}_{010}$ monopole mode which is characterized by a pure longitudinal electric field being maximum on the beam axis and decreasing with higher distance to the beam axis. The magnetic field of the mode is axially symmetric, transversal to the electric field and zero at the beam axis.

The major design parameters as well as simulation and measurement results are summarized in Tab. 1. The shuntimpedance of the kicker cavities was determined from the longitudinal distribution of the electric field along the beam axis [60] which was measured by using a bead pull perturbation method [73]. Taking into account one $200 \mathrm{~W}$ amplifier, the measured attenuation of the rf cables and the used rf splitters, the maximum achievable accelerating voltage is $(198 \pm 22) \mathrm{V}$ for cavity 1 and $(191 \pm 21) \mathrm{V}$ for cavity 2 for the setup shown in Fig. 31 .

\subsubsection{Transverse stripline kicker}

Corresponding to the bandwidth and frequency range of transverse multi-bunch dipole oscillations a broadband stripline kicker is used as correction element for the transverse bunch-by-bunch feedback system. The operation frequency ranges from DC to $f_{\mathrm{rf}} / 2$ and defines the basic layout of the kicker. The kicker is based on a coaxial design of a four quadrant waveguide, matched to a line impedance of $50 \Omega$. Figure 35 shows a cut through the kicker structure. Eight specially designed rf feedthroughs

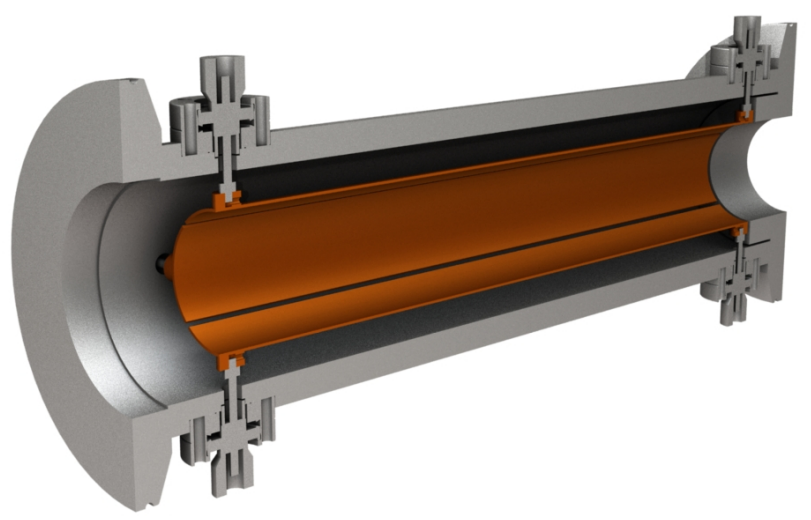

Figure 35. Cut through the stripline kicker used at ELSA [70].

connect the inner copper striplines to the outer shell and are used to inject a signal to the waveguide structure. The signal propagates across the striplines, is extracted on the opposite side and terminated in a matched rf load. The impedance matching of the kicker structure allows for affecting only a single bunch with a calculated and amplified correction signal. A $180^{\circ}$ splitter is used to split the amplified correction signal into two equal parts with opposite polarity. Each signal drives one of both opposing 


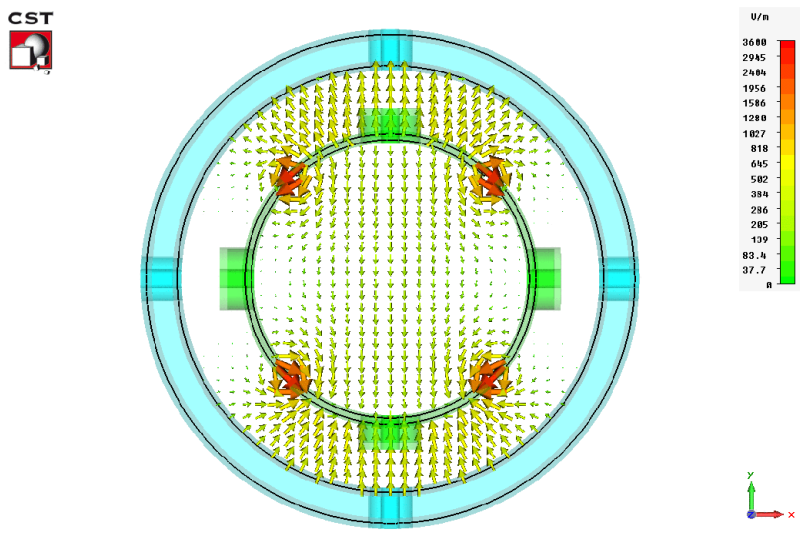

Figure 36. Electric dipole field distribution of the stripline kicker in vertical operation calculated with CST Microwave Studio $^{\circledR}[59]$.

striplines, which yields to an electric dipole field in the center of the kicker structure corresponding to Fig. 36.

The shunt impedance $R_{S}$ of a stripline kicker with line impedance $Z_{L}$ depends on excitation frequency $f$, length $L$ and radius $r$ of the striplines which cover an angle of $\theta$ as [78]

$$
R_{S}(f)=\frac{8 Z_{L} c^{2}}{\pi^{2} r^{2} f^{2}} \sin ^{2}\left(\frac{\theta}{2}\right) \sin ^{2}\left(\frac{2 \pi f}{c} \cdot L\right)
$$

Thus, the length of the striplines defines the bandwidth of the kicker structure. At ELSA, a minimum bandwidth of $250 \mathrm{MHz}$ is required for damping transverse dipole bunch oscillations. Setting the length of the striplines to $30 \mathrm{~cm}$ ensures a proper kicking strength over the full bandwidth, as well as a vanishing shunt impedance on the rf harmonics. With this ansatz, the excitation and consequently the heating and beamloading of the kicker structure is minimized. The shunt impedance measurement results for the ELSA stripline kicker are shown in Fig. 37.

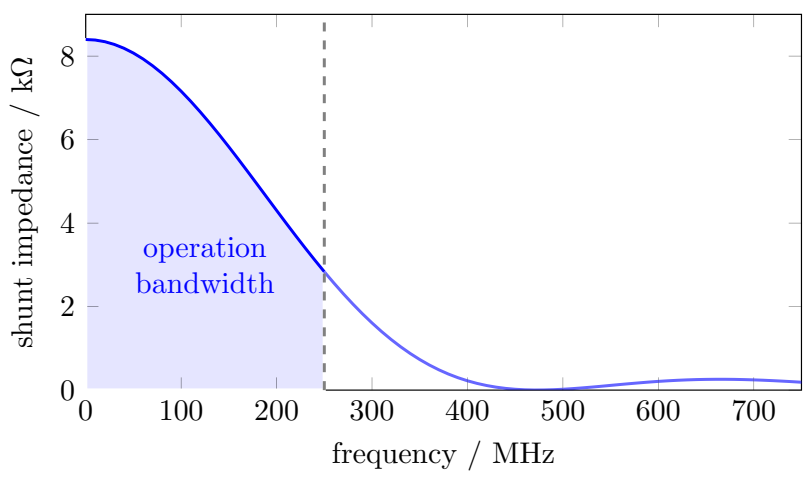

Figure 37. Shunt impedance of the ELSA stripline kicker as function of the excitation frequency [70].

\subsubsection{Feedback performance}

\section{Grow-damp measurement and Fourier spectrum}

To verify the damping capability of the bunch-by-bunch feedback system, so-called grow-damp measurements are of high importance. For that purpose, the system is deactivated for a few milliseconds using a filter with zero gain and an internal software trigger of the DSP unit, resulting in a growth of the bunches' oscillation amplitude in case of non-sufficient synchrotron radiation damping. After the reactivation of the standard filter, the impact of the feedback system on the oscillating bunches can be observed immediately.

Exemplary, such a measurement is shown in Fig. 38 which was conducted after the successful commissioning of the longitudinal feedback system at a beam energy of $2.35 \mathrm{GeV}$ and for a stored beam current of about $20 \mathrm{~mA}$ in the storage ring. The digital input data of the longitudinal feedback system for bunch number 162 is presented, as its oscillation amplitude was largest among all filled buckets. The data was recorded turn-by-turn by the ADC of the DSP module resulting in a sampling frequency of $1.8236 \mathrm{MHz}$. Above noise ${ }^{3}$ an exponential growth of the oscillation amplitude can be observed which confirms again that longitudinal coherent oscillations are already present at the storage ring for relatively moderate beam currents. After activation of the feedback system at $5 \mathrm{~ms}$, the reduction of the amplitude within $300 \mu$ s and the damping performance of the system are clearly visible.

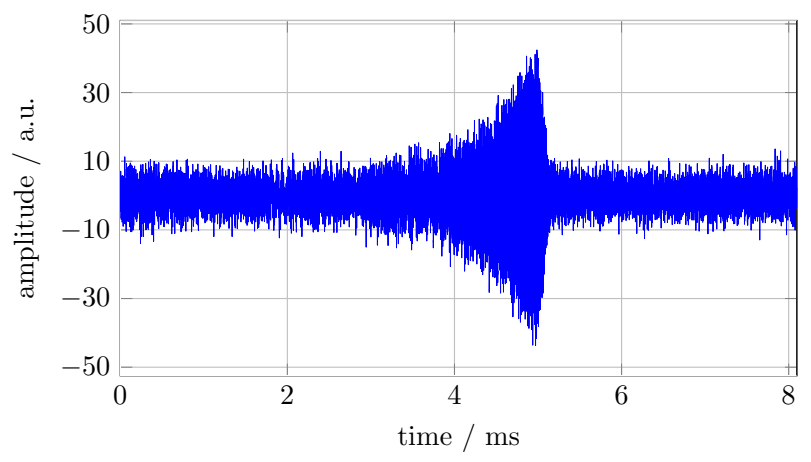

Figure 38. Grow-damp measurement for bunch number 162 at a beam energy of $2.35 \mathrm{GeV}$ after the commissioning of the longitudinal feedback system at the storage ring: At $0 \mathrm{~ms}$ the feedback system was deactivated, at $5 \mathrm{~ms}$ the system was activated again. The fast damping performance of the system is observable [62].

Furthermore, with the Fourier spectrum of such a grow-damp measurement the resistive damping of the feedback system can be verified. First, the Fourier spectrum for each filled bucket is obtained by computing the discrete Fourier transform for each of the bunches' digitized amplitudes. By averaging over all single Fourier spectra, the Fourier spectrum for the complete grow-damp measurements is calculated. In case of the presented example, the Fourier spectrum is shown in Fig. 39.

\footnotetext{
${ }^{3}$ The noise level of the recorded ADC data amounts to $\pm 10 \mathrm{ADC}$ counts which is also measurable without electron beam.
} 


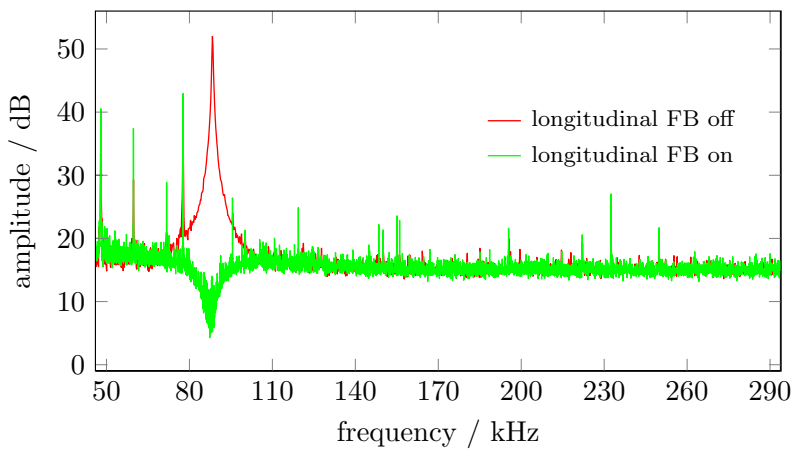

Figure 39. Fourier spectrum of the grow-damp measurement presented in Fig. 38: Peak and notch at nearly the same frequency of about $88 \mathrm{kHz}$ indicate a perfect resistive damping of the longitudinal feedback system. The additional narrow peaks are caused by noise signals and are also visible in spectra recorded without beam [62].

Due to the successful damping of the oscillations of all bunches the peak disappears completely and converts to a notch whose depth can be used as a measure for the quality of the damping performance. The notch is a consequence of the additional suppression of noise signals by the feedback system in the range of the synchrotron frequency, which is caused by the strong beam response on resonance. In addition, no shift between the frequencies of peak and notch can be observed, which indicates a purely resistive damping and overall a perfectly working system.

\section{Operation on the fast energy ramp}

In view of the phase characteristics of the used FIR filters in the DSP units, it is essential that the longitudinal and transverse tunes are kept constant during the energy ramp. Otherwise, a shift of the synchrotron or betatron frequencies would result in a change of the filter's phase and the corresponding phase of the generated correction signal. In this case, the primarily realized resistive damping performance of the feedback system exists no longer and in the worst case, if the phase shift exceeds $90^{\circ}$, an excitation of coherent electron oscillations can occur. To ensure matched feedback settings and stable operation independent of beam energy and operation mode of the storage ring, a new LLRF system has been installed which is capable of stabilizing and controlling amplitude and phase of the acceleration voltage (see Section 3.5).

A successful operation of the feedback system can be monitored best by measuring the Fourier spectra of each filled bucket on the fast energy ramp. As already mentioned the presence and depth of a notch in the spectra at the synchrotron and betatron frequencies indicates the quality of the damping performance. From the changing positions of the notches, the accuracy of the stabilization of the longitudinal and transverse tunes can be verified.

Figure 40 shows the time series of Fourier spectra for the longitudinal and transverse planes successively recorded every $20 \mathrm{~ms}$ on the fast energy ramp. In this measurement, a beam current of about $35 \mathrm{~mA}$ has been ac-

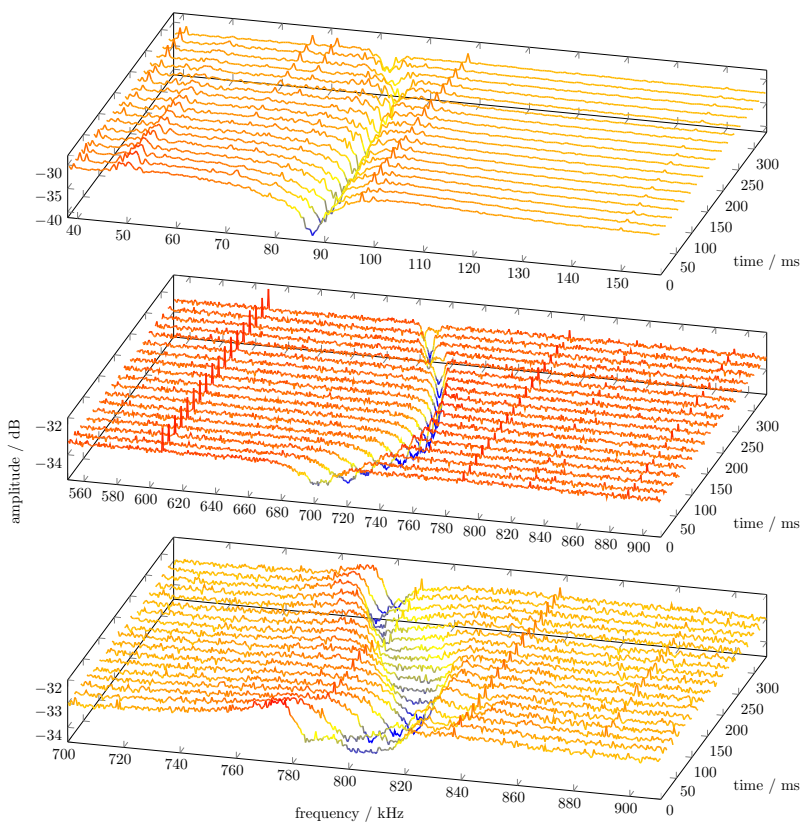

Figure 40. Feedback operation on the fast energy ramp. A beam current of about $35 \mathrm{~mA}$ has been accelerated from $1.2 \mathrm{GeV}$ to $3.2 \mathrm{GeV}$ with a ramp speed of $6 \mathrm{GeV} / \mathrm{s}$. Fourier spectra for all three planes have been recorded successively every $20 \mathrm{~ms}$. From top to bottom: longitudinal plane, horizontal plane, vertical plane. [70]

celerated from $1.2 \mathrm{GeV}$ to $3.2 \mathrm{GeV}$ with a ramping speed of $6 \mathrm{GeV} / \mathrm{s}$. The characteristic suppression (notch) at the synchrotron and betatron frequencies is clearly visible. In the longitudinal plane, the position of the notch and therefore the damping is almost independent of energy, which demonstrates a successful stabilization of the longitudinal tune. Only at the end of the energy ramp the synchrotron frequency is slightly reduced which is caused by a lack of acceleration voltage due to missing rf power required for compensating the beam loading in the accelerating cavities. The situation is different for the transverse planes, where fast varying magnetic fields caused by eddy currents in the vacuum chamber lead to additional magnetic multipoles which affect the beam focussing and therefore the transverse tunes. To allow an effective compensation of these effects, a dedicated tune measurement and correction procedure has been developed based on the experience with the feedback system and using its diagnostic capabilities. It is presented in Section 3.6.

\subsection{RF amplitude and phase stabilization}

To ensure matched feedback settings and stable operation independent of beam energy and operation mode of the storage ring, a new LLRF system has been installed. It is capable of stabilizing and controlling amplitude and phase of the acceleration voltage.

\section{Low level rf system}

The new LLRF9/500 system, developed and manufactured by Dimtel, Inc. [74] has been successfully commissioned 


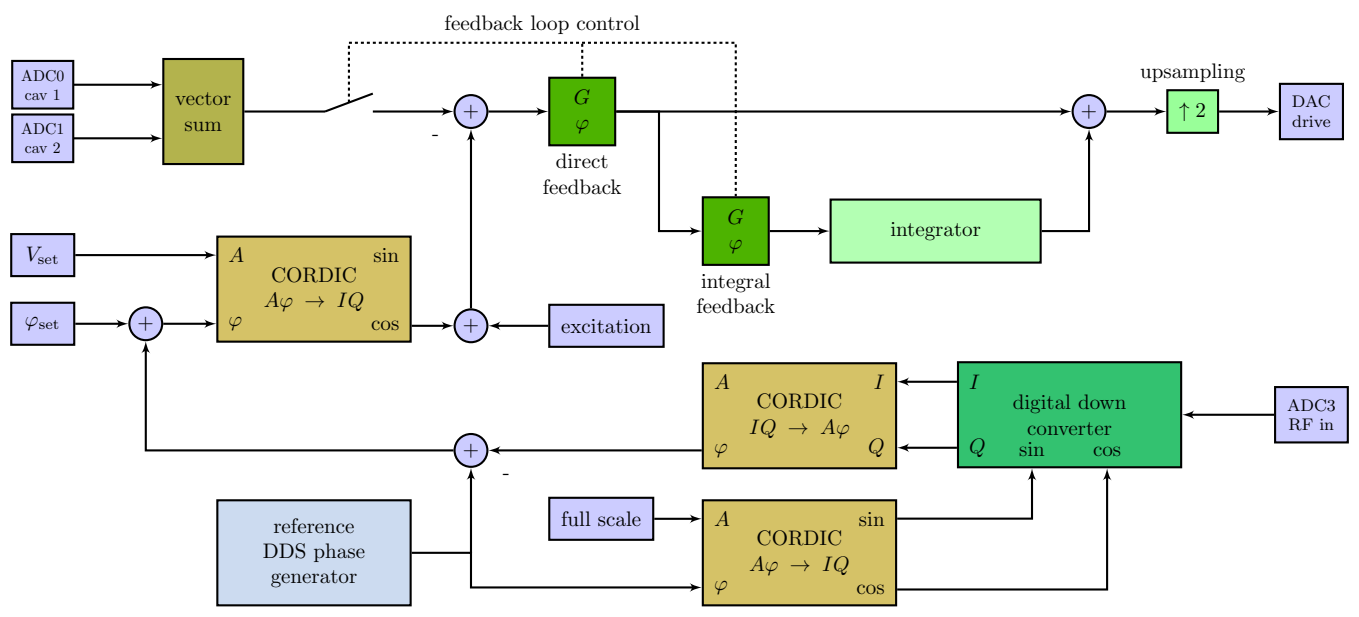

Figure 41. Overview of the signal processing of the LLRF system [70].

at ELSA. Nine wideband rf inputs are used to monitor $\mathrm{rf}$ cavity and waveguide signals. Two cavity probe signals are used in a digital feedback loop to stabilize the vector sum of the accelerating cavity voltages. The LLRF system is based on a fully digital approach using an FPGA. All of the nine analog rf inputs are digitized after downconversion to $1 / 12$ of the rf frequency ( $42 \mathrm{MHz})$ and then processed by the FPGA. The cavity field stabilization is performed by two feedback loops: the low-latency proportional and the integral loop. The detailed layout and performance of the FPGA feedback processing is described in $[57,64]$, an overview of the signal processing is given in Fig. 41.

A digital proportional and integral feedback loop on the cavity station voltage and phase allows for compensation of the voltage fluctuations due to beam-loading effects. The fully digital approach provides monitoring capability of amplitude and phase for each input signal with $10 \mathrm{~Hz}$ update frequency. The FPGA continuously compares all input signals with the adjustable interlock thresholds. With the intermediate frequency of $42 \mathrm{MHz}$ the maximum latency for detecting an interlock event is limited to 96 ns. Every interlock event is latched and time stamped. Fast data acquisition with pre-trigger capture provides information for post-mortem analysis. The FPGA generates a trip signal which, together with an external interlock input, controls an rf switch disabling the klystron drive output. The new LLRF system implements adjustment of the two tuner motors per accelerating cavity with a PID controller, operating at $10 \mathrm{~Hz}$. The tuner loop acts to keep the cavity probe and forward signals in phase for minimum reflected power. In addition to stabilize the amplitude and phase, the LLRF system allows to ensure a constant synchrotron frequency during the fast energy ramp via hardware triggerable ramp profiles for amplitude and phase setpoints.

\section{Beam dynamics on the energy ramp}

On the fast energy ramp, the synchrotron frequency $f_{s}$ of ultra-relativistic electrons oscillating longitudinally with respect to the synchronous phase $\varphi_{s}$ is mainly given by the accelerating voltage $U$, the mean energy loss per turn $e U_{\text {rev }}$, and the beam energy $E[79]$ :

$$
f_{s}=f_{\mathrm{rev}} \sqrt{\frac{e \alpha_{c} h}{2 \pi} \frac{U}{E} \sqrt{1-\frac{U_{\mathrm{rev}}^{2}}{U^{2}}}},
$$

where $\alpha_{c}$ represents the momentum compaction factor, $f_{\text {rev }}$ the revolution frequency and $h$ the harmonic number. The revolution voltage is determined by

$$
U_{\mathrm{rev}}=\frac{e \beta^{3}}{3 \varepsilon_{0}\left(m_{0} c^{2}\right)^{4}} \frac{E^{4}}{R}
$$

which defines the acceleration voltage required to compensate for the energy loss of the electrons due to synchrotron radiation in the circular accelerator. A constant synchrotron frequency can be achieved by adjusting

$$
\sqrt{U^{2}-U_{\mathrm{rev}}^{2}}=\frac{f_{s}^{2}}{f_{\mathrm{rev}}^{2}} \frac{2 \pi E}{e \alpha_{c} h}
$$

which leads to an energy dependent accelerating voltage which has to be set to

$$
U(E)=\sqrt{U_{\mathrm{rev}}^{2}+E^{2} \frac{f_{s}^{4}}{f_{\mathrm{rev}}^{4}} \frac{4 \pi^{2}}{e^{2} \alpha_{c}^{2} h^{2}}} .
$$

The synchronous phase is then changing by

$$
\Delta \varphi_{s}(E)=\sin ^{-1}\left(\frac{U_{\mathrm{rev}}}{U(E)}\right)
$$

which has to be compensated by shifting the rf phase setpoint value accordingly on the fast energy ramp.

An example for a fast energy ramp profile of the $\mathrm{rf}$ station is shown in Fig. 42. Here, the synchrotron frequency of $92 \mathrm{kHz}$ is stabilized on the energy ramp from $1.2 \mathrm{GeV}$ to $3.2 \mathrm{GeV}$ applying a ramp profile of 512 steps within about $333 \mathrm{~ms}$ and changing the accelerating voltage from 1.1 MV to 3.1 MV. Applying a phase ramp with a total amplitude of $-15.2^{\circ}$ allows for compensation of the change of synchronous phase and ensures a matched timing of the bunch-by-bunch feedback loop. 


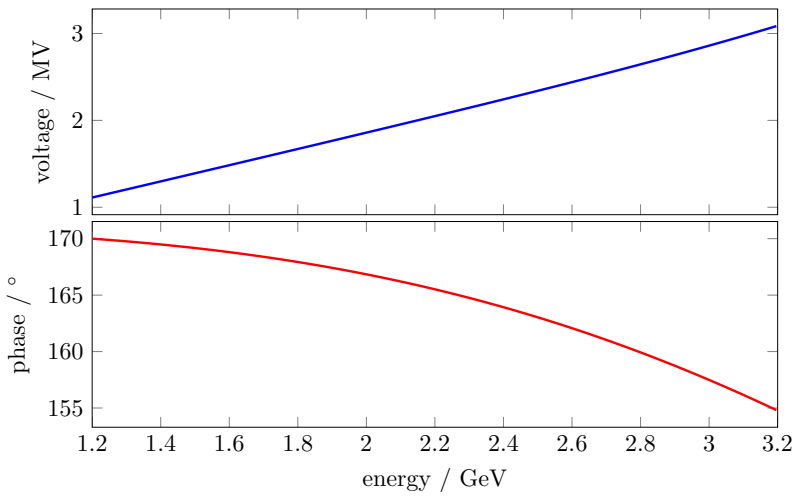

Figure 42. Required settings of the accelerating voltage and phase on the fast energy ramp from $1.2 \mathrm{GeV}$ to $3.2 \mathrm{GeV}$ to achieve a constant beam phase and synchrotron frequency of $92 \mathrm{kHz}[70]$.

\subsection{Fast tune measurement and correction}

Fast ramping of magnets like dipoles and quadrupoles requires precise timing and correction techniques for magnet strengths. This has been achieved by measuring horizontal and vertical betatron tunes with a fast tune monitor and applying feed forward tune corrections to the fast energy ramp. The tune is measured using the bunch-by-bunch feedback's data acquisition capability and beam excitation functionality. Applying an offline FFT and data evaluation allows extracting tunes and calculating tune shifts as a correction signal for the main magnets' current ramp profiles. This is used as well for precise tune correction required by the bunch-by-bunch feedback system and to prevent polarized electron beams from depolarization on the fast energy ramp.

\subsubsection{Requirements on the tune measurement}

The tunes $Q_{x, z}$ of a circular accelerator cannot be chosen arbitrarily. Due to optical resonances the beam's oscillation amplitude may be driven to grow exponentially. This will cause beam loss or reduce the beam's quality for user operation. These resonances occur if the following condition is fulfilled:

$$
j \cdot Q_{x}+k \cdot Q_{z}=N \quad \text { with } j, k, N \in \mathbb{N} .
$$

In the ELSA storage ring the horizontal tune is placed close to the third integer optical resonance $Q_{x}=4 \frac{2}{3}$ and is slowly shifted towards it, allowing slow beam extraction. From this it is evident that the betatron tunes should be determined with accuracy better than one percent.

\section{Timing mismatch of magnet ramps}

Applying a fast energy ramp of $6 \mathrm{GeV} / \mathrm{s}$ requires a precise setting of the storage ring's magnets' current ramp profiles and timings. For this purpose, a hardware ramp-start trigger is applied to all of the magnets' power supplies which starts the programmed ramp. Every timing mismatch of the dipole and quadrupole ramps will cause transverse tune shifts, which can cause partial or complete beam loss when crossing an optical resonance. Such timing errors of the storage ring's main magnets' power supplies have been investigated to be in the order of 5 milliseconds. To successfully eliminate those timing errors and to stabilize the corresponding tunes, a tune measurement with temporal resolution of about $1 \mathrm{~ms}$ is required.

\section{Tune shifts induced by eddy currents in vacuum chambers}

A fast changing magnetic field, which is used to generate the fast energy ramp of the storage ring, induces voltages in the conducting vacuum chambers installed inside the dipole and quadrupole magnets. These induced voltages lead to eddy currents which generate additional magnetic field contributions and act on the beam's motion. Amongst other effects, this will lead to transverse tune shifts, generated by additional quadrupole strengths. To correct for those tune shifts a tune measurement and correction scheme with a temporal resolution well below the ramping time of up to $330 \mathrm{~ms}$ is needed.

\subsubsection{Setup of the measurement}

The fast tune measurement requires a fast readout of bunch position data as well as data evaluation and tune extraction. This fast readout has been realized using the bunchby-bunch feedback system described in Section 3.4. To extract the beam's oscillation frequency from the raw data of a single bunch, a Fast Fourier Transform (FFT) is applied while exciting the corresponding bunch using a frequency sweep over the expected betatron frequency range. The excitation is generated by the bunch-by-bunch feedback system and applied by the rf kickers.

First, a drive signal is applied to one single bunch. It is set up to sweep over a fixed frequency range centered at the corresponding tune's frequency. The time of the frequency sweep has to be lower than the resulting FFT window to ensure a complete frequency scan over the chosen frequency span. After reading out the raw ADC data from the feedback system's single bunch acquisition buffer, the positions of the bunch collected every turn are split into subsets including a fixed number of turns each. The number of turns corresponding to the length of those subsets is chosen to match the desired temporal step size of the tune measurement settings.

For every subset an FFT is performed to extract the corresponding amplitude spectrum of the beam's motion. Searching for the maximum amplitude in every spectrum allows the extraction of the single bunch's tune in every subset.

\subsubsection{First results}

In order to correct for arbitrary tune and timing shifts on the fast energy ramp, the ELSA control system provides an interface for a feed forward method for tune correction. First, a delay $\Delta t_{F, D}^{0}$ can be added to the start of the magnetic field ramp of the focussing $(F)$ and defocussing $(D)$ quadrupoles to correct for timing errors. The remaining 
tune shifts along the energy ramp can be reduced by applying corrected quadrupole strengths which are based on the tune measurement. Using the accelerator model of the control system, the tune shifts are mapped into correction currents for every time step. The resulting current ramps are send to the power supplies. This procedure allows for iterating over several measurements to enhance the accuracy of the resulting tune correction.

The measurements at the ELSA storage ring have been conducted using a temporal resolution of $N=2000 \mathrm{rev}$ olutions, corresponding to $\Delta t=1.096 \mathrm{~ms}$. The resulting frequency resolution is given by

$$
\Delta f=\frac{f_{\text {rev }}}{N} \approx 0.91 \mathrm{kHz},
$$

which corresponds to $\Delta Q=5 \times 10^{-4}$. Frequency and tune resolution match the required accuracies.

\section{Optimizing timing shifts on the fast energy ramp}

To reduce the transverse tune shifts on the fast energy ramp, the timing delays of the focussing and defocussing quadrupole power supplies have been changed and the resulting tune shifts were measured. The minimum vertical tune shift is achieved by setting the delay of the defocussing quadrupoles to $\Delta t_{D}^{0}=1.5 \mathrm{~ms}$ and $\Delta t_{F}^{0}=$ $-0.25 \mathrm{~ms}$ for the horizontal tune by shifting the focussing quadrupoles' ramp. Figure 43 shows the behavior of the vertical and horizontal tunes during the first $160 \mathrm{~ms}$ of the fast energy ramp with and without optimized delay. The negative shift has been achieved by shifting dipole and defocussing quadrupole timing setpoints to positive values.

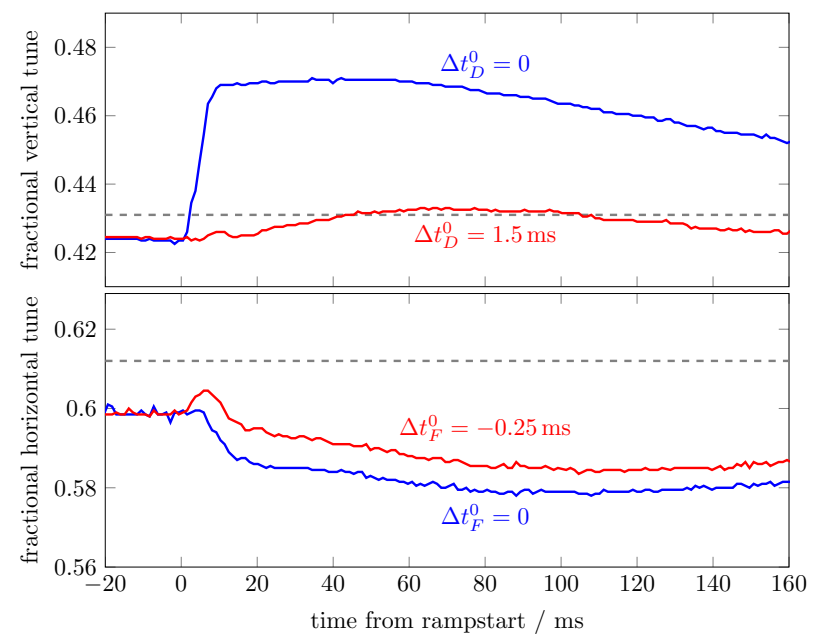

Figure 43. Measurement of the fractional tune for different timing shift corrections of the quadrupoles [70].

\section{Tune Correction on the fast energy ramp}

To achieve a successful and reliable operation of the bunch-by-bunch feedback system and to facilitate the correction of intrinsic depolarizing resonances applying fast tune jumps, both horizontal and vertical tunes have to be corrected to match the setpoints. Figure 44 shows a measurement of the vertical tune spectrum during the fast energy ramp, including four typical tune jumps. The sawtooth like tune jumps with a fall time of a few milliseconds are clearly visible, demonstrating the performance of the fast tune measurement. Since the transverse tune is corrected only during the fast energy ramp, it does not match its setpoint before and after the fast energy ramp. This leads to a minor tune deviation at ramp-start and rampstop. In future operation, a new ramp profile generator will allow to correct the tunes at the energy-flattop as well. This would prevent the observed tune deviation which potentially leads to beam loss.

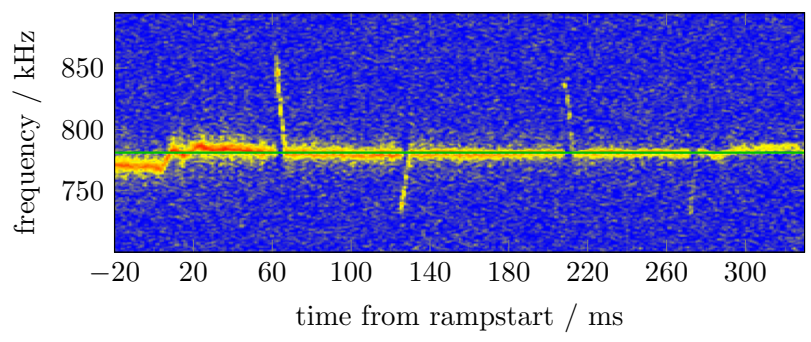

Figure 44. Corrected vertical tune on fast energy ramp from $1.2 \mathrm{GeV}$ to $3.2 \mathrm{GeV}$ when operating with polarized electrons and applying four tune jumps. The green line shows the setpoint of the vertical tune [70].

\subsection{Visualization of beam dynamics}

The emission of visible synchrotron radiation allows the imaging of electron beam dynamics through camera systems. At ELSA a streak camera ${ }^{4}$ functions as fast time resolving beam diagnostic apparatus, capable of visualizing dynamics in all three dimensions from milliseconds down to the picoseconds time regime. A designated synchrotron radiation diagnostics beamline was installed [66, 85] and commissioned (Fig. 45) in order to provide up to singleshot 3D images of the revolving electron bunches in the storage ring. The system is capable of bunch length and damping time measurements as well as monitoring coherent and incoherent beam dynamics.

\subsubsection{Experimental setup}

Streak cameras are specialized on measuring longitudinal photon distributions with the consequence of suppressing spatial information of one transverse plane. The ELSA beamline includes an optics set-up which preserves both transverse dimensions by splitting the photon beam (see Fig. 46) and transversally rotating one branch with a Dove prism. An adjustable path length provides a controllable photon time-of-flight delay for the second branch. Interchangeable magnification optics, a photon time-of-flight delay line and motorized mirrors allow proper signal positioning on the streak camera's sensitive measuring area.

\footnotetext{
${ }^{4}$ Hamamatsu C10910
} 


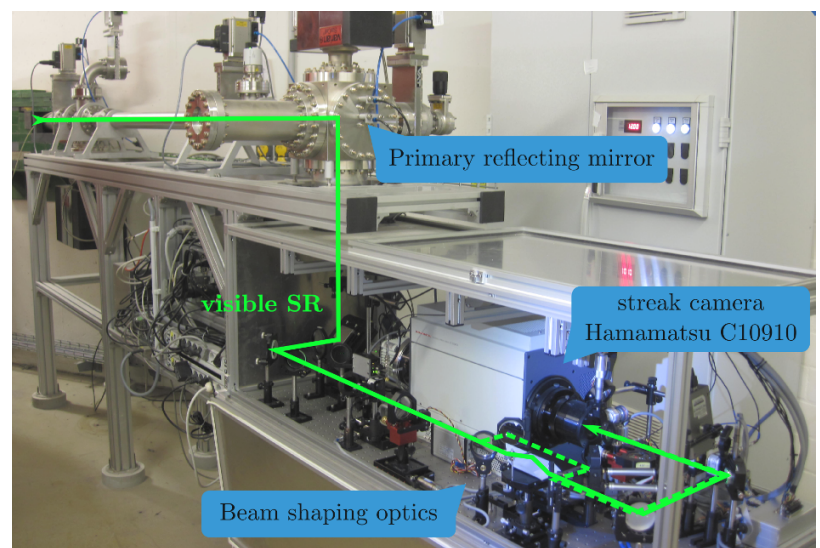

Figure 45. Synchrotron radiation diagnostics beamline with streak camera housing outside of the ELSA shielding tunnel [69].

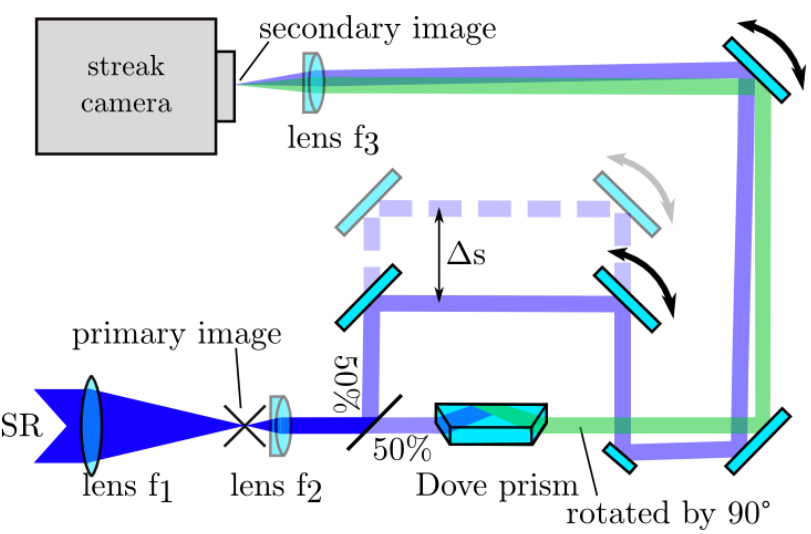

Figure 46. A horizontal and vertical beam image is coupled onto the streak camera to provide full 3D imaging capability [69].

\subsubsection{D bunch imaging capability}

As the intensity of synchrotron radiation scales with the electron current, the observation of instabilities in the high-current regime yields sufficient single-shot images at shortest time scales. Figure 47 shows multiple single-shot electron bunch length measurements in top and side view, where the vertical axis denotes the longitudinal photon distribution. Due to the synchroscan measuring technique every fourth bunch is displayed in one row (e.g. bunches no. 1 and no. 5 in the top row and no. 3 and no. 7 in the bottom row). In addition, the dual imaging creates another pair of rows with vertical displacement $\Delta t_{\mathrm{ph}}$ corresponding to the time of flight difference. On the horizontal axis the bunches are $8 \mathrm{~ns}$ apart, whereas the horizontal and vertical set differ by an arbitrary distance set by the optics. At $80 \mathrm{~mA}$ of stored beam current and $1.2 \mathrm{GeV}$ beam energy the storage ring chromaticity was increased by a factor $>10$, creating an instability affecting only a selection of electron bunches (e.g. no. 1). The result is a longitudinal deformation as indicated by the measured bunch length. As the sextupole component was further increased, more bunches were successively affected in the same manner, eventually resulting in complete beam loss. Stan-

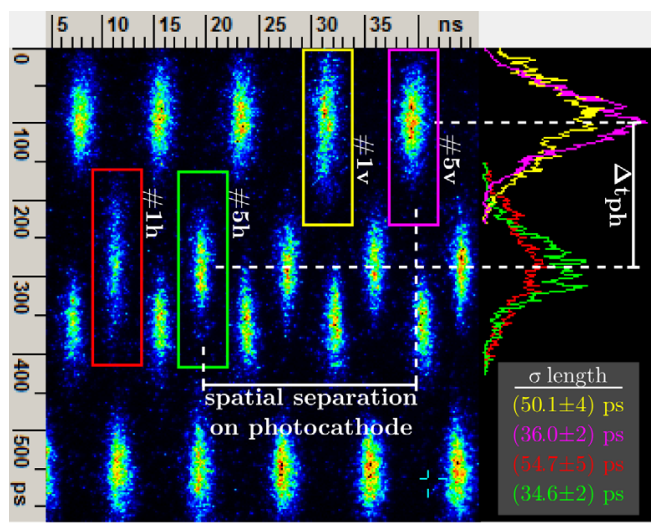

Figure 47. Single shot image of multiple electron bunches in top (h) and side view (v). A beam instability causes bunch lengthening for particular bunches before complete beam loss occurs. The actual electron beam dimension is larger horizontally than vertically despite the appearance in the image which is caused by an aperture restriction in the optical beamline [69].

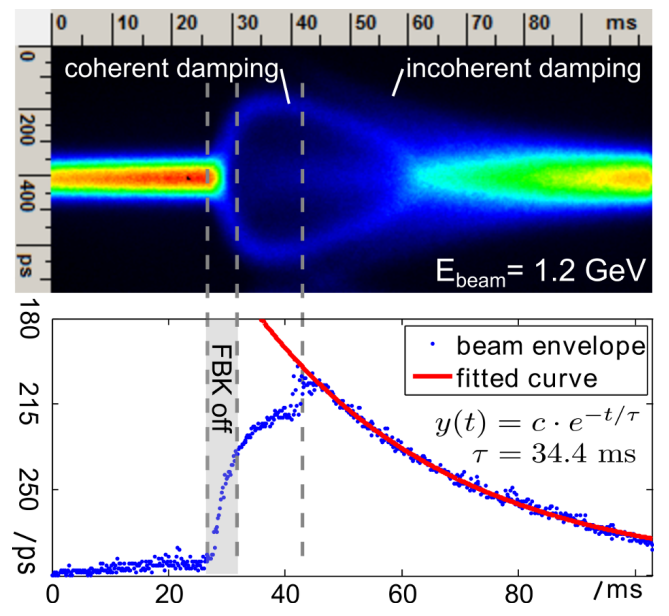

Figure 48. Grow-damp measurement of coherent and incoherent synchrotron oscillations. The streak camera is the only device capable of measuring incoherent oscillation amplitudes [67].

dard ELSA operation indicates no such instability behavior. However, future machine settings, which may cause abnormal intra-bunch dynamics whose traces result in intensity distortions as e.g. in head-tail instabilities, can now be identified.

\subsubsection{Multi-bunch beam dynamics}

Beam dynamics up to time scales of several milliseconds can be observed in the same manner as described in Section 3.7.2. However, individual bunches cannot be resolved transversally but overlap within a single horizontal signal line containing only longitudinal information. Figure 48 illustrates a grow-damp measurement, where the bunch-by-bunch feedback system (see Section 3.4) is turned off for $5 \mathrm{~ms}$ at a beam current of $145 \mathrm{~mA}$. Immediately multi-bunch instabilities start to grow resulting in coherent longitudinal particle oscillations which are damped 
by the feedback system after it is turned on again. As the decohered particle population is not affected by the feedback correction, natural damping occurs due to the emission of synchrotron radiation. The exponential decay of the envelope can be evaluated from the bitmap image.

Cases could be identified where the feedback correction is not powerful enough to regenerate a stable beam. For example, Fig. 49 shows reoccurring grow-damp behavior as function of the accelerating cavities' temperature. As the re-excitation period peaks near $36.8^{\circ} \mathrm{C}$, it vanishes for decreasing or increasing temperatures [68]. However, the amplitude and envelope shape describing the decoherence trace differs for higher or lower temperatures.

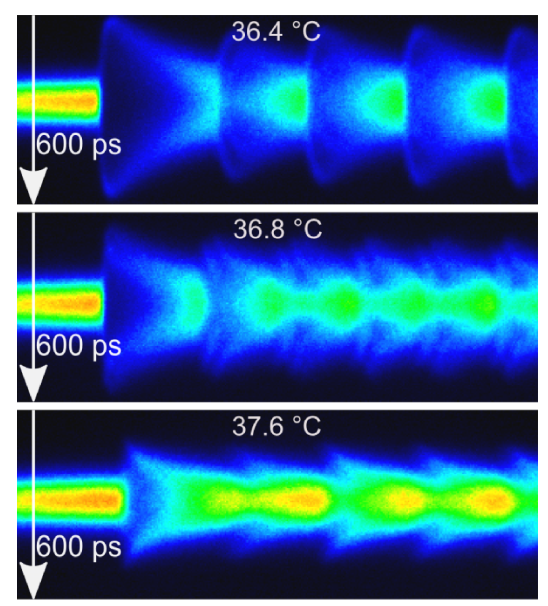

Figure 49. Multi-bunch instabilities reoccurring for certain accelerating cavity temperatures [68].

\section{Slow beam extraction}

A quasi continuous (but still bunched) external electron beam is produced using the technique of resonant beam extraction. To achieve a constant external beam current, an intensity feedback is applied generating appropriate current ramps for the extraction quadrupoles. The required small shift of the storage ring's horizontal tune causes a slowly changing beam pointing which has to be precisely monitored and compensated. The emittance of the external beam depends on the settings of the storage ring's optics and shows minor variations during the extraction period.

\subsection{Resonant beam extraction technique}

The slow beam extraction is based on an accelerator operation close to a third integer stop band. Dedicated sextupole magnets located in dispersion free straight sections of the storage ring are powered in order to excite a third-order horizontal betatron resonance. As a consequence, the electrons' motions in the horizontal phase space become unstable except for those located in a small central part which is bound by separatrices (the stable triangle, see Fig. 50). The area of the central part depends on the strength of the

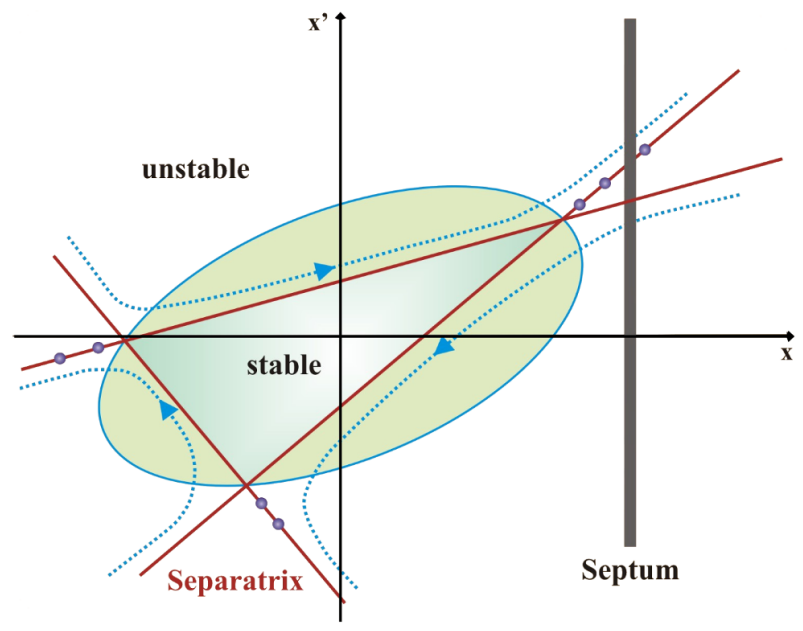

Figure 50. Particles' motion in the horizontal phase space when operating the accelerator close to a third-order resonance. The area of the stable triangular central part depends on the distance from the resonance and the resonance strength. Unstable particles will spiral outwards and will be extracted when passing the septum of the extraction magnet.

sextupole fields and the distance of the tune from the thirdorder resonance. By shrinking the area of the stable triangle, parts of the electron beam become unstable, resulting in an increasing horizontal betatron amplitude. These unstable electrons move along the separatrix lines in phase space and are finally extracted after crossing the septa of two extraction magnets [1].

\subsection{Position and intensity stabilization}

Due to the required variation of the stable phase area, the positions of the separatrix lines are shifted during the extraction period. This in general causes a small shift of the angle of the extracted beam which increases during the extraction period. A compensation method commonly applied at proton accelerators aligning all extraction separatrices (known as Hardt condition [80]) cannot be realized at ELSA due to missing flexibility of the storage ring's optics. Instead, the beam deflection in the first septum magnet is changed appropriately, thus compensating the shift of angular displacement of the extracted beam at the position of the septum blade to first order. The required additional septum control is derived adding a scalable fraction of the extraction quadrupole current setting to the current setpoint of the septum magnet's power supply. The scaling factor is determined empirically by minimizing the shift of the external beam at the tagging systems of the nuclear physics experiments. This procedure requires precise beam position monitoring at the photon tagger which is performed by a newly developed non-invasive monitor system based on two rf diagnostic cavities. This system is described in detail in [81].

A constant external beam current over the extraction period is achieved by applying a feedback loop to the extraction quadrupoles. Either the overall tagging rate or the voltage signal of an rf diagnostic cavity serving as beam 
intensity monitor (see [81, 82]) can be selected as input for a PI controller of the quadrupole power supplies.

\subsection{Emittance measurements}

The emittance measurements are based on recording the transverse intensity distribution of the electron beam with the help of luminescence screens and synchrotron radiation monitors. For this purpose, dedicated systems have been set up at the storage ring and the external beam lines (see [65]).

\subsubsection{Measurement principle}

Once knowing the beam's relative momentum deviation $\Delta p / p$, the beta functions $\beta_{x, z}$ and the dispersion $D_{x}$ at the position of the monitor, the horizontal and vertical emittance can be extracted from

$$
\begin{aligned}
& \sigma_{x}(s)=\sqrt{\epsilon_{x} \cdot \beta_{x}(s)+\left(D_{x}(s) \cdot \frac{\Delta p}{p}\right)^{2}} \text { and } \\
& \sigma_{z}(s)=\sqrt{\epsilon_{z} \cdot \beta_{z}(s)}
\end{aligned}
$$

where the rms widths $\sigma_{x}$ and $\sigma_{z}$ are determined from fitting a Gaussian function to the measured transverse intensity profiles. Whereas $\Delta p / p$, which is energy dependent, can be taken from the accelerator's optics model, $\beta_{x}, \beta_{z}$ and $D_{x}$ depend on the extraction optics and therefore have to be measured.

In a first step the dispersion function is determined by varying the $\mathrm{rf}$ frequency $f_{\mathrm{rf}}$ and measuring the corresponding shifts $\Delta x$ of the entire beam:

$$
D(s)\left(-\frac{1}{\alpha_{c}} \frac{\Delta f_{\mathrm{rf}}}{f_{\mathrm{rf}}}\right)=\Delta x(s) .
$$

This measurement is repeated for different quadrupole strengths, and a dispersion parameter $D_{0}$ and its derivative $D_{0}^{\prime}$ at a position in front of the first quadrupole of the external beamline are calculated from this data.

In a second step, the emittances $\epsilon_{x}$ and $\epsilon_{z}$ are determined applying the quadrupole scan technique. Here, intensity profiles are recorded for different settings of a focussing quadrupole in the external beamline and an optics dependent function is fitted to the set of extracted rms beam widths, where contributions caused by the dispersion are subtracted according to Eq. 4. This procedure allows the extraction of both, the beta function and the emittance (for further details see e.g. [83, 84]) and has been repeated for different optics settings of the storage ring.

\subsubsection{Results}

To determine the impact of the storage ring optics on the emittance of the extracted beam, two sets of measurements have been carried out: First, when increasing the strength of the extraction sextupoles and thereby increasing the excitation of the third integer resonance, the step size at the septum blade becomes larger leading to an increase of the external beam's horizontal emittance (see

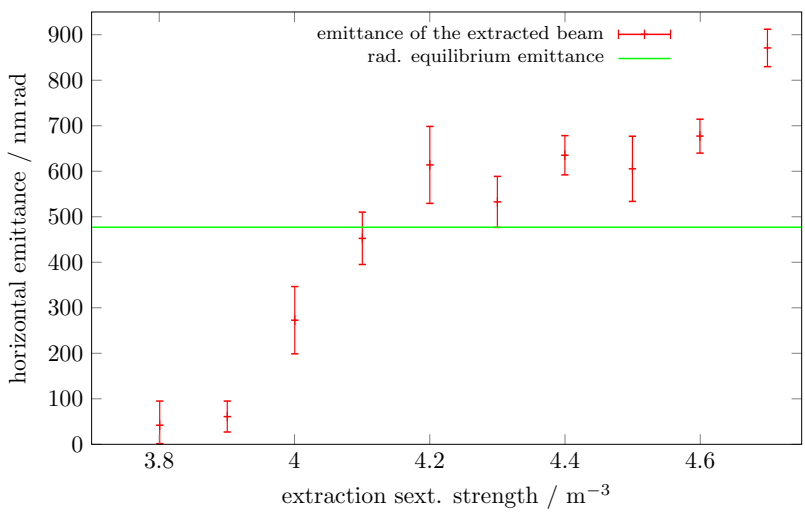

Figure 51. Measured horizontal beam emittance as function of the extraction sextupole strength for a beam energy of $2.35 \mathrm{GeV}$. The green line indicates the radiative equilibrium emittance in the storage ring.

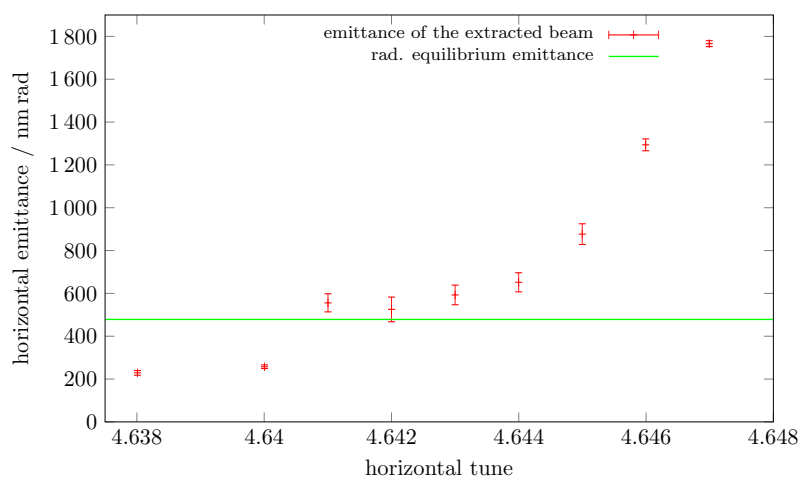

Figure 52. Measured horizontal beam emittance as function of the horizontal tune for a beam energy of $2.35 \mathrm{GeV}$. The green line indicates the radiative equilibrium emittance in the storage ring.

Fig. 51). Second, when increasing the strength of the extraction quadrupoles and thereby shifting the horizontal betatron tune closer to the third integer stop band, again the step size at the septum blade becomes larger leading to an increase of the external beam's horizontal emittance (see Fig. 52).

In addition, time resolved measurements for fixed settings of the storage ring's optics have been performed. Here, the temporal change of the external beam's emittances has been determined and compared to the emittances of the stored beam which have been measured simultaneously (see [84]). Whereas the horizontal emittance is mainly determined by the (fixed) step size at the septum blade and therefore remains constant throughout the extraction period (see Fig. 53), the vertical emittance depends on the coupling of the horizontal and vertical betatron oscillations. The extracted electrons originate from the unstable region in phase space and therefore have larger betatron amplitudes than the stored electrons occupying the central stable triangle. The vertical emittance of the extracted beam is therefore slightly larger than the vertical emittance of the stored beam (see Fig. 54). 


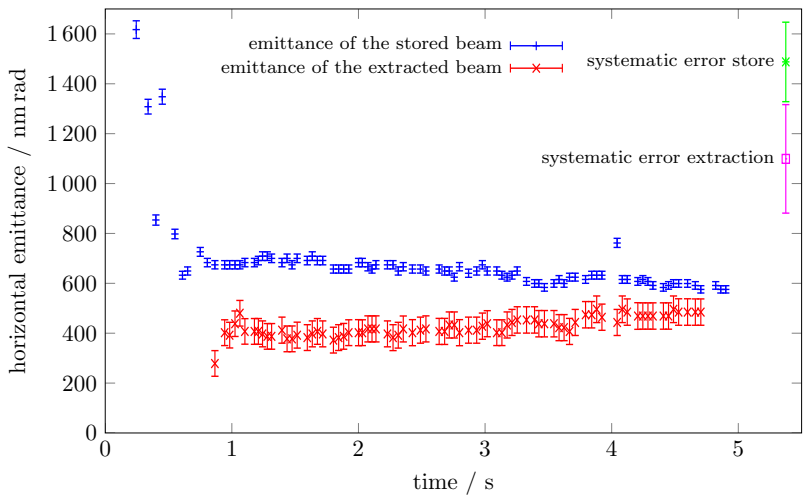

Figure 53. Measured temporal change of the horizontal beam emittance over the extraction period. The extraction of a $2.35 \mathrm{GeV}$ beam started at $1 \mathrm{~s}[85]$.

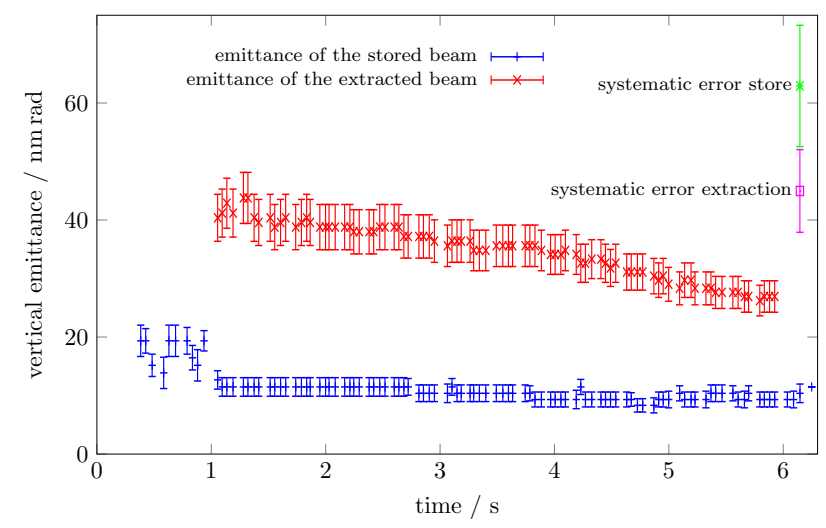

Figure 54. Measured temporal change of the vertical beam emittance over the extraction period. The extraction of a $2.35 \mathrm{GeV}$ beam started at $1 \mathrm{~s}$ [85].

\section{Energy increase}

Regarding the production of linearly polarized photon beams, an increase of the maximum achievable electron beam energy is highly desirable. Whereas circularly polarized photons, generated by polarization transfer from longitudinally polarized electrons in the bremsstrahlung process, get maximum polarization in case of a full energy transfer from electrons to photons [86], the achievable linear photon polarization via coherent bremsstrahlung drops significantly at high energy transfers (see e.g. [5]) and vanishes for a full energy transfer. In order to optimize the overall figure of merit (in which the photon polarization enters quadratically), the kinematics of the coherent bremsstrahlung are typically chosen such that the coherent peak is set at about half of the electron beam energy. This limits the useful energy range of linearly polarized photons to the same value and makes an increase of the electron beam energy highly attractive.

The maximum beam energy at the ELSA storage ring is mainly limited by the achievable magnetic bending fields and acceleration voltage. In principle, an energy upgrade may be attained by reducing the gap of the dipole magnets and the installation of superconducting rf cavi- ties. Design concepts and the impact on beam dynamics and cavity control at the fast ramping ELSA storage ring have been investigated for a proposed energy increase to $5 \mathrm{GeV}$ and are presented in the following.

\subsection{Magnets and beam dynamics}

\subsubsection{Installation of new magnets}

To generate the required bending field of $1.53 \mathrm{~T}$, the existing dipole magnets have to be replaced by new ones. The gap has to be reduced from $50 \mathrm{~mm}$ to $38 \mathrm{~mm}$, the number of windings of the coils must be increased from 14 to 20. In addition, the existing dipole power supply has to be modified with respect to the higher total resistance of the magnet coils, the requirements on the current will not change.

The operating point in the tune diagram is fixed to the settings used up to now in order to achieve a dispersion suppression in the straight sections caused by the missing magnet concept of the ELSA lattice. The existing quadrupole and sextupole magnets can be operated up to $5 \mathrm{GeV}$ without modifications. Only minor modifications of the quadrupole power supplies (increase of the maximum current from 910 A to 970 A because of the energy dependence of the quadrupole strength) are required.

No modification of the kicker magnets of the injection system is needed. The extraction magnets ( 2 septum magnets) are limited in field strength to a maximum beam energy of $3.5 \mathrm{GeV}$ and have to be replaced by stronger ones.

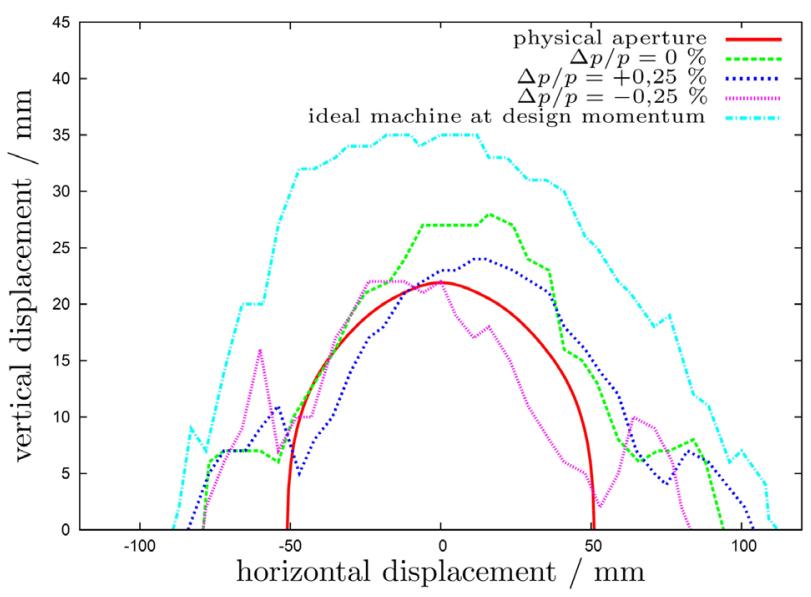

Figure 55. Dynamic aperture of the ELSA storage ring with alignment and magnetic field errors influenced by eight sextupole magnets for chromaticity correction: For each line in the plot several error distributions have been simulated in order to obtain sufficient statistics. The minimum value of those apertures was selected and is shown here.

\subsubsection{Static aperture and beam lifetime}

The accelerator lattice was investigated up to $5 \mathrm{GeV}$ beam energy. It turned out that the aperture limitations (except 


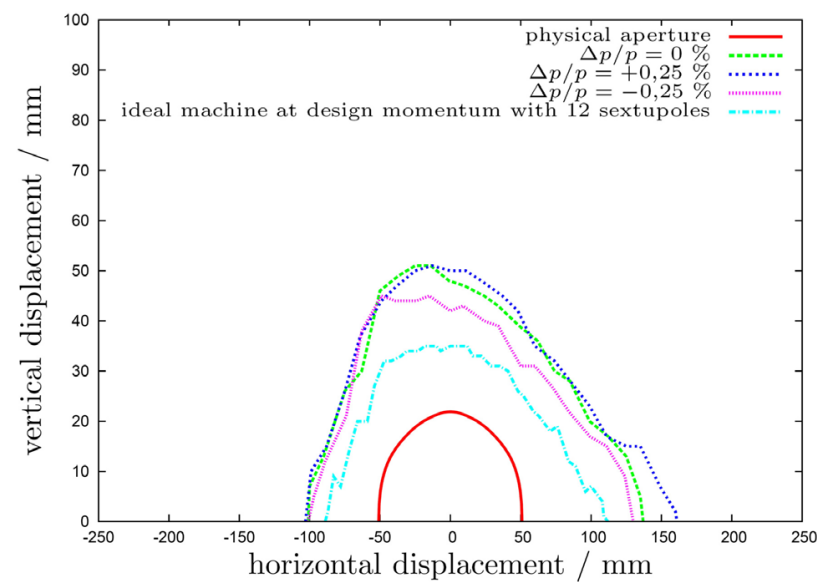

Figure 56. Dynamic aperture of the ELSA storage ring with alignment and magnetic field errors using twelve sextupole magnets for chromaticity correction. In this case larger magnetic field errors would be acceptable than the ones used in Fig. 55.

for the extraction septa which have to be replaced for reasons mentioned above) of the existing vacuum chambers allow for a beam lifetime of about 50 hours which is several orders of magnitude larger than the requirements for accelerator operation with beam extraction to external experiments.

\subsubsection{Dynamic aperture}

Detailed investigations of the influence of alignment and field errors of the dipole and quadrupole magnets as well as higher multipole components were performed applying particle tracking simulations with the help of dedicated software packages. From these calculations we derived the dynamic aperture which indicates the maximum beam sizes circulating stably without significant beam loss. Even in case of relative momentum deviations of $0.25 \%$ - i.e. twice the beam energy spread at $5 \mathrm{GeV}$ - and compensation of chromatic errors, the existing number of sextupoles seems sufficient in order to obtain a dynamic aperture comparable with the physical one (see Fig. 55).

The situation changes in case of larger multipole contributions which may arise from partial saturation of the magnets at maximum energy. In the worst case this would require an installation of additional four sextupole magnets for chromaticity correction (see Fig. 56). This can be decided only if new dipole magnets are available, so that they can be analyzed regarding their magnetic field errors.

\subsection{Operation of superconducting cavities}

The accelerator operation for hadron physics experiments requires beam life times of at least one minute at flat top energy in order to avoid significant beam losses. This corresponds to an overvoltage factor of 2.69 and a peak accelerating voltage of $13.67 \mathrm{MV}$ which has to be generated. An appropriate $500 \mathrm{MHz}$ accelerating structure is given by the geometry of the superconducting (sc) five-cell cavities

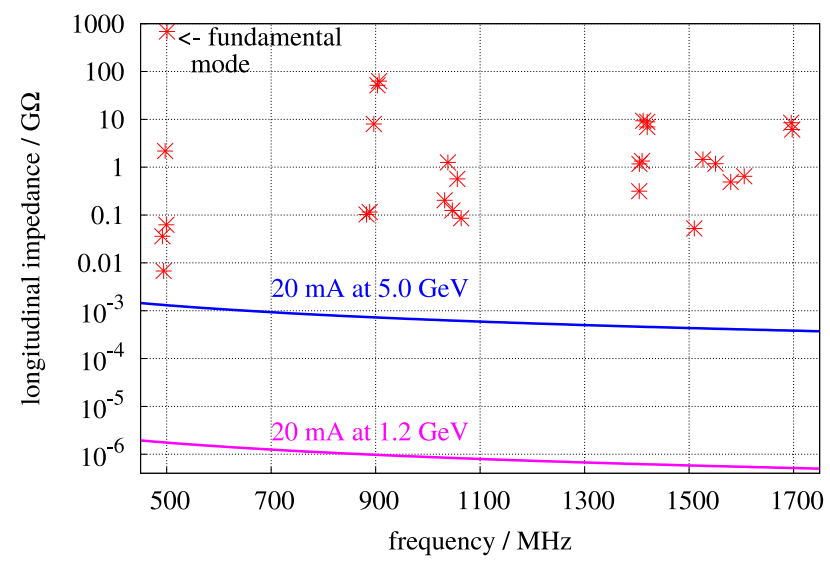

Figure 57. Longitudinal shunt impedances of monopole HOMs and typical threshold impedances for longitudinal multi-bunch instabilities at a beam current of $20 \mathrm{~mA}$ in the storage ring [87].

constructed for the JAERI FEL linac by the former company Accel Instruments in the early '90s [88]. These sc acceleration modules are characterized by an operating gradient of up to $5 \mathrm{MV} / \mathrm{m}$ with a cavity quality factor above $10^{9}$. Using two of these five-cell cavities, the necessary overall cavity voltage of $13.67 \mathrm{MV}$ could be provided.

\subsubsection{Cavity coupling and detuning}

Assuming a shunt impedance of $500 \mathrm{G} \Omega$ and a quality factor of $2 \times 10^{9}$, the beam current dependent optimal coupling factor of the cavities was calculated [87]. It turned out that, using this setup and the existing rf plant $(250 \mathrm{~kW}$ klystron, waveguides, etc.), an internal beam with up to $50 \mathrm{~mA}$ beam current can be accelerated up to $5 \mathrm{GeV}$ and stored for at least some seconds with negligible beam loss (which is sufficient in case of slow beam extraction). The coupling would be adjusted to an optimal coupling factor of 2720 for $50 \mathrm{~mA}$ at $5 \mathrm{GeV}$ and does not need to be varied for different beam currents and beam energies.

The cavity detuning defines the difference between the rf frequency and the resonance frequency of a cavity and has to be adjusted during the beam injection and on the energy ramp by fast frequency tuning of the cavities. The maximum cavity detuning was calculated to be less than $16 \mathrm{kHz}$, the maximum detuning speed is less than about $32 \mathrm{kHz} / \mathrm{s}$ [87] which should be achievable using state-ofthe-art tuning devices for sc resonators. A typical scenario of an accelerator cycle is illustrated in Fig. 58.

\subsubsection{HOM and beam instabilities}

The investigation of the required higher order mode (HOM) damping was based on the geometry of the JAERI structure. All relevant monopole and dipole HOMs were simulated numerically with the software package CST Microwave Studio $^{\circledR}$ neglecting the HOM damping of the main power coupler [87]. Figures 57 and 59 show the results for the longitudinal and transversal impedances of 


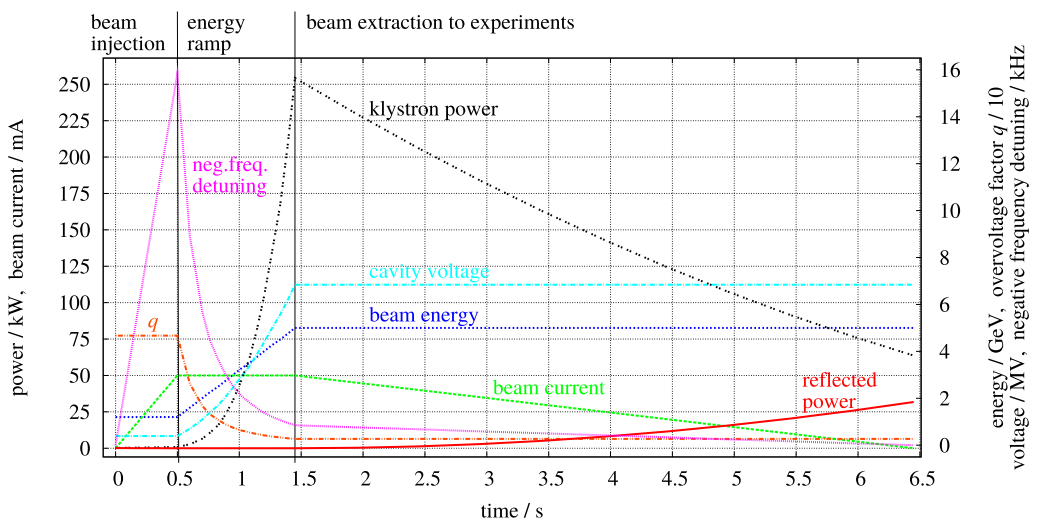

Figure 58. Accelerator cycle for a beam current of $50 \mathrm{~mA}$ up to $5 \mathrm{GeV}$ using two superconducting cavities with a shunt impedance of $5 \times 10^{11} \Omega$ and a quality factor of $Q_{0}=2 \times 10^{9}$. Time dependence of cavity voltage and overvoltage factor, total klystron power as well as reflected power and negative frequency detuning per cavity are shown [87].

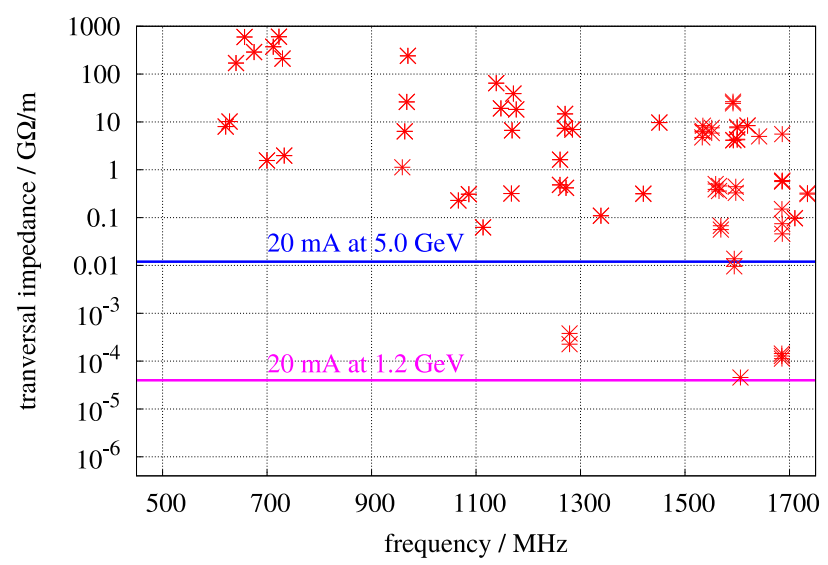

Figure 59. Transversal shunt impedances of dipole HOMs and typical threshold impedances for transverse multi-bunch instabilities at a beam current of $20 \mathrm{~mA}$ in the storage ring [87].

the HOMs and typical threshold impedances. It turned out that the growth rate of all higher order monopole modes and a large number of dipole modes are well above the stability threshold given by radiative damping and may excite coherent beam instabilities. Therefore sophisticated HOM couplers have to be developed, even in case of partial damping of HOMs by the fundamental mode coupler. The layout of these HOM couplers is considered to be the major problem of a possible future energy upgrade program.

\section{Summary}

Within the past 12 years, the electron stretcher accelerator facility ELSA has been successfully upgraded with respect to beam polarization, intensity and stability. The upgrade program comprised developments and various dedicated measures for an increase of beam current and energy, damping of coherent beam instabilities, compensation of depolarizing resonances and enhancement of the quality of the extracted beam. The envisaged intensity increase of approximately a factor of 10 has been successfully achieved, and polarization losses on the fast energy ramp have been reduced to less than $10 \%$ up to energies of about $3 \mathrm{GeV}$. The quality and stability of the extracted beam is sufficient for a reliable generation of linearly and circularly polarized photon beams for photoproduction experiments. ELSA is therefore well prepared for a successful continuation of the proposed hadron physics experimental program.

The work reported here would not have been possible without the support from the Deutsche Forschungsgemeinschaft within the SFB/TR16.

\section{References}

[1] W. Hillert, Eur. Phys. J. A 28 s1, 139 (2006)

[2] J. Hartmann et al., Phys. Lett. B 748, 212 (2015)

[3] B. Bantes et al., Int. J. Mod. Phys. Conf. Ser. 26, 1460093 (2014)

[4] A. Bella et al., EPJ Web of Conf. 81, 06002 (2014)

[5] D. Elsner et al., Eur. Phys. J. A 39, 373 (2009)

[6] W. Hillert et al., AIP Conf. Proc. 570, 961 (2001)

[7] H. Dutz et al., Phys. Rev. Lett. 91, 192001 (2003)

[8] H. Dutz et al., Phys. Rev. Lett. 93, 032003 (2004)

[9] H. Dutz et al., Phys. Rev. Lett. 94, 162001 (2005)

[10] D. Heiliger et al., PSTP'09 Conf. Proc., 232 (2010)

[11] D. Heiliger et al., J. Phys. Conf. Ser. 298, 012003 (2011)

[12] D. Heiliger et al., IPAC'12 Conf. Proc., 1521 (2012)

[13] D. Heiliger et al., IPAC'13 Conf. Proc., 294 (2013)

[14] D. Heiliger et al., IPAC'11 Conf. Proc., 1123 (2011)

[15] W. Hillert et al., IPAC'11 Conf. Proc., 2133 (2011)

[16] A. Balling et al., PAC'09 Conf. Proc., 3714 (2009)

[17] A. Balling et al., AIP Conf. Proc. 1149, 809 (2009)

[18] J.-P. Thiry et al., IPAC'12 Conf. Proc., 789 (2012)

[19] J.-P. Thiry et al., IPAC'13 Conf. Proc., 479 (2013)

[20] J.-P. Thiry et al., IPAC'14 Conf. Proc., 1111 (2014)

[21] O. Boldt et al., PAC'11 Conf. Proc., 1507 (2011) 
[22] J.F. Schmidt et al., IPAC'14 Conf. Proc., 1108 (2014)

[23] M. Hoffmann et al., AIP Conf. Proc. 570, 756 (2001)

[24] O. Boldt et al., IPAC'11 Conf. Proc., 2130 (2011)

[25] J.F. Schmidt et al., IPAC'13 Conf. Proc., 891 (2013)

[26] J.F. Schmidt et al., IPAC'15 Conf. Proc., 808 (2015)

[27] J.F. Schmidt et al., IPAC'16 Conf. Proc., 695 (2016)

[28] W. Hillert et al., AIP Conf. Proc. 1149, 1160 (2009)

[29] R. Koop et al., IPAC'12 Conf. Porc., 783 (2012)

[30] R. Koop et al., IPAC'16 Conf. Porc., 95 (2016)

[31] A. Septier, Focusing of Charged Particles (Acad. Press, New York, 1967)

[32] W.B. Hermannsfeldt, SLAC-PUB 331, UC28 (1988)

[33] D. Heiliger, Diss., Univ. Bonn (2014)

[34] M. Yamada et al., Appl. Surf. Sci. 70/71, 531 (1992)

[35] V.E. Andreev et al., AIP Conf. Proc. 570, 901 (2000)

[36] M. Baylac et al., Phys. Rev. ST Accel. Beams 8, 123501 (2005)

[37] M. Yamada and Y. Ide., Surf. Sci. 339, L914 (1995)

[38] P. Tomkiewicz et al., Appl. Surf. Sci. 252, 7647 (2006)

[39] O.M. Katz and E.A. Gulbransen., Rev. Sci. Instrum. 31, 615 (1960)

[40] U. Bischler and E. Bertel, J. Vac. Sci. Technol. A 11, 458 (1993)

[41] V. Bargmann et al., Phys. Rev. Lett. 2, 435 (1959)

[42] M. Froissart and R. Stora, Nucl. Instr. and Meth. 7, 297 (1960)

[43] A Dieckmann et al., IPAC'12 Conf. Proc., 3623 (2012)

[44] O. Boldt et al., IPAC'12 Conf. Proc., 1326 (2012)

[45] C. Sanderson and R. Curtin, J. Open Sour. Soft. 1, 26 (2016)

[46] M. Borland, Advanced Photon Source LS-287, (2000)

[47] J. F. Schmidt, github.com/janfschmidt/palattice

[48] MAD-X, Methodical Accelerator Design, https:// mad.web.cern.ch/mad/

[49] J. F. Schmidt, www.ctan.org/pkg/tikz-palattice

[50] A. A. Sokolov and I. M. Ternov, Soviet Physics Doklady 8, 1203 (1964)

[51] Ya. S. Derbenev and A. M. Kondratenko, Sov. Phys. JETP 37, no. 6, (1973)

[52] S. Agostinelli et al., Nucl. Instr. Meth. A 506, 250 (2003)

[53] F. Klarner et al., LINAC'08 Conf. Proc., 392 (2009)
[54] F. Klarner et al., LINAC'10 Conf. Proc., 325 (2010)

[55] S. Mey et al., IPAC'11 Conf. Proc., 1072 (2011)

[56] M. Schedler et al., LINAC'14 Conf. Proc., 847 (2014)

[57] D. Sauerland et al., IPAC'13 Conf. Proc., 2717 (2013)

[58] A. Roth et al., IPAC'11 Conf. Proc., 250 (2011)

[59] M. Schedler et al., IPAC'11 Conf. Proc., 481 (2011)

[60] N. Heurich et al., IPAC'11 Conf. Proc., 484 (2011)

[61] M. Schedler et al., IPAC'12 Conf. Proc., 2840 (2012)

[62] A. Roth, Diss., Univ. Bonn (2013)

[63] M. Schedler et al., IPAC'13 Conf. Proc., 2714 (2013)

[64] M. Schedler et al., IPAC'14 Conf. Proc., 2418 (2014)

[65] S. Zander et al., PAC'11 Conf. Proc., 408 (2011)

[66] S. Zander et al., IPAC'12 Conf. Proc., 795 (2012)

[67] M. Switka et al., IPAC'14 Conf. Proc., 3471 (2014)

[68] M. Switka et al., IBIC'14 Conf. Proc., 144 (2014)

[69] M. Switka et al., IPAC'15 Conf. Proc., 144 (2015)

[70] M. Schedler, Diss., Univ. Bonn (2015)

[71] H. Alberto, Dipl., TU Dortmund (1998)

[72] CST, Computer Simulation Technology, http://www. cst.com/

[73] L.C. Maier Jr. and J.C. Slater, J. Appl. Phys. 23, 68 (1952)

[74] Dimtel Inc., San Jose, USA, www.dimtel.com

[75] Y. Kim et al., IEEE Trans. Nucl. Sci. 47, no. 2, 452 (2000)

[76] W.Z. Wu et al., Nucl. Instr. Meth. Phys. Res. A 632, 32 (2011)

[77] R. Boni et al., EPAC'96 Conf. Proc, 1881 (1996)

[78] D.A. Goldberg and G.R. Lambertson, AIP Conf. Proc. 249, 537 (1992)

[79] H. Wiedemann, Particle accelerator physics 2 nd ed. (Springer, Berlin, 2003)

[80] W. Hardt, CERN PS-DL-LEAR-Note, 81-6 (1981)

[81] T. Pusch et al., Phys. Rev. ST Accel. Beams 15, 112801 (2012)

[82] T. Pusch et al., DIPAC'11 Conf. Proc., 263 (2011)

[83] S. Zander et al., IPAC'11 Conf. Proc., 1168 (2011)

[84] S. Zander et al., IPAC'12 Conf. Proc., 792 (2012)

[85] S. Zander, Diss., Univ. Bonn (2013)

[86] H. Olsen and L.C. Maximon, Phys. Rev. 114, 887 (1959)

[87] A. Roth et al., IPAC'11 Conf. Proc., 253 (2011)

[88] M. Pekeler et al., APAC'07 Conf Proc., 791 (2007) 